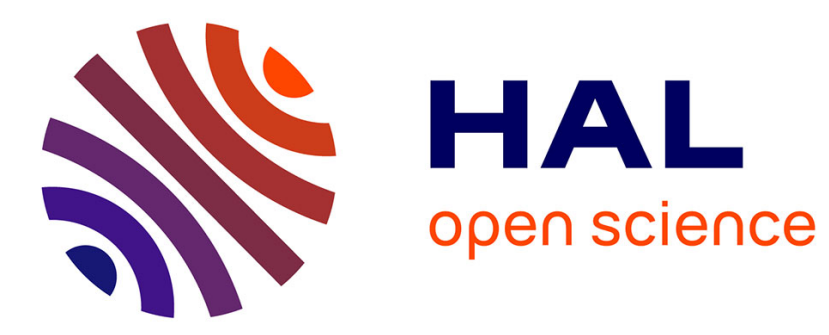

\title{
Multiscale Modeling of Follicular Ovulation as a Mass and Maturity Dynamical System \\ Philippe Michel
}

\section{To cite this version:}

Philippe Michel. Multiscale Modeling of Follicular Ovulation as a Mass and Maturity Dynamical System. Multiscale Modeling and Simulation: A SIAM Interdisciplinary Journal, 2011, 9, pp.282 313. 10.1137/090745738 . hal-01236197

\section{HAL Id: hal-01236197 \\ https://hal.science/hal-01236197}

Submitted on 1 Dec 2015

HAL is a multi-disciplinary open access archive for the deposit and dissemination of scientific research documents, whether they are published or not. The documents may come from teaching and research institutions in France or abroad, or from public or private research centers.
L'archive ouverte pluridisciplinaire HAL, est destinée au dépôt et à la diffusion de documents scientifiques de niveau recherche, publiés ou non, émanant des établissements d'enseignement et de recherche français ou étrangers, des laboratoires publics ou privés. 


\title{
MULTISCALE MODELING OF FOLLICULAR OVULATION AS A MASS AND MATURITY DYNAMICAL SYSTEM*
}

\author{
PHILIPPE MICHEL ${ }^{\dagger}$
}

\begin{abstract}
During each ovarian cycle, numerous follicles are in competition for their survival, only a few of them ovulate and participate to the reproduction as fertilizable oocyte. Therefore, the development of ovarian follicles is crucial for the survival of mammals species and a better understanding of this selection process is required to improve the treatment of infertility or to improve the reproduction potential in domestic species. In this work, we introduce the system of nonlinear PDEs which modelize the growth and maturation of ovarian follicles. Then we study the dynamic of this system of nonlinear PDEs using analysis and dynamical systems tools.
\end{abstract}

Key words. Dynamical System, Bifurcation, Catastrophe, PDE, Asymptotic behavior

AMS subject classifications. 35Q92, 35B40, 35B32, 37Fxx, 37Gxx

1. Introduction. Reproduction ability and efficiency are major assets in the natural selection of species. It is studied to improve the reproduction potential of cattle or to treat the infertility in the human researches (see [28, 23, 22]). Even if the infertility seems to be due for 40 percent to the male and 40 percent to the female (10 percent both and 10 percent without any reason), treatments and studies of infertility are mostly focused on female infertility [22, 23, 36, 24, 14].

During each menstrual cycle, eggs are developed. They are the culmination of a growth and maturation process of ovarian follicles which are, in some sense, the basic unit of female reproductive biology. Ovarian follicle is composed of roughly spherical aggregations of cells (granulosa) found in the ovary and contain a single oocyte. In the ovarian cycle (growth, i.e., cell division and maturation process), numerous follicles are in competition for their survival (see [3, 4, 5, 6, 14, 13, 21, 24, 28, 22]). Only a few number of them ovulate and participate to the reproduction as fertilizable oocyte(s), the others degenerate (atresia) (see [5, 14, 12]). Therefore, the development of ovarian follicles is crucial for the survival of mammals species and this cell-scale competition process leads to a specie-scale competition process through the reproduction [14, 13]. Thus, a better understanding of this selection process is required to improve the treatment of infertility or to improve the reproduction potential in domestic species.

The follicular development result from a complex process involving the Follicle Stimulating Hormone (FSH) which controls the proliferation rate, the apoptosis rate and the differentiation rate of follicles. The follicles secrete hormones such as estradiol exerting a negative feedback on FSH release. At the end of this process, the survival follicles are those who are the less sensitive to the fall of plasmatic level of FSH in other words the most mature follicles, the others undergo atresia.

In this work, we are interested in two models of the ovarian follicles development. In the first one, H. M. Lacker [21] considers that follicles are defined by their maturity which follow a dynamical system of coupled Ordinary Differential Equations. The assumptions, done by the authors, are mostly imputable to mathematical considerations. In the second one [14], proposed by the INRIA team Sisyphe, follicles are defined by the density of their cells, structured by their age $a$ and maturity $\gamma$,

\footnotetext{
*This work was supported by REGATE - INRIA project

${ }^{\dagger}$ Université de Lyon, UMR 5208, Institut Camille Jordan ; Ecole Centrale de Lyon, 36 avenue Guy de Collongue - 69134 Ecully Cedex - FRANCE (philippe.michel@ec-lyon.fr). Questions, comments, or corrections to this document may be directed to that email address.
} 
which follows a system of coupled Partial Differential Equations of conservation laws.

The multiscale model of follicle growth and maturation process. We introduce more specifically the multiscale model [14] :

- Cell division. In each follicle, at the beginning of the growth and maturation process, we observe that cells divide and participate to the growth of the follicle. After some times, cells stop to divide. We know that cells which divide follow a mitosis cycle in four phases (G1-S-G2-M). More precisely, the age $a$ is a marker of progression in the cell cycle in the G1 (beginning of the cell cycle) and S to M (mitosis, i.e., cell division) phases of the cell cycle. The duration of the G1 phase is $a_{1}>0$ and the total cycle duration is $a_{2}>a_{1}$. The reason of the end of the mitosis of cells is their ability to use the FSH and the maturity marker $\gamma \in\left[0, \gamma_{\max }\right]$ is used to sort the cycling and noncycling cells by comparison to a threshold $\gamma_{s}$ and to characterize the cell vulnerability towards apoptosis ${ }^{1}[3,4,5,6,14,13,21]$.

- Definition of the age/maturity speed rate $g_{f}, h_{f}$ and death rate $\Lambda$. The velocities of aging $g_{f}$ and maturation $h_{f}$ of cells as well as the death rate of cells $\Lambda$ (apoptosis rate) depends on the mean maturity of the follicle $f: \mathcal{M}_{f}$

$$
\mathcal{M}_{f}=\iint_{\mathcal{S}} \gamma \Phi_{f} d a d \gamma
$$

and of $\mathcal{M}_{T}$ the total maturity of the system (all the follicles)

$$
\mathcal{M}_{T}=\sum_{f \in\{\text { Follicles }\}} \mathcal{M}_{f}
$$

where $\Phi_{f}(t, a, \gamma)$ is the cell density function of the follicule $f$ and

$$
\mathcal{S}:=\left[0, a_{2}\right] \times\left[0, \gamma_{\max }\right] \bigcup\left[0, \infty\left[\times\left[\gamma_{s}, \infty[,\right.\right.\right.
$$

through a local control $u_{f}=u_{f}\left(\mathcal{M}_{f}, \mathcal{M}_{T}\right)$ which represents intrafollicular bioailable FSH level and the mean maturity of all the follicles through a global control $U=\left(\mathcal{M}_{T}\right)$ which can be interpreted as the FSH plasmatic level. For instance, in paper [14], the authors let the age speed rate

$$
g_{f}\left(\gamma, u_{f}\right)=\tau_{g f}\left(1-g_{1} \omega_{s-}(\gamma)\left(1-u_{f}\right)\right)
$$

where $\gamma \mapsto \omega_{s-}(\gamma)$ is a "smooth indicator function" (see [14]) : nearly constant equal to 1 when $\gamma<\gamma_{s}$ and vanishes when $\gamma>\gamma_{s}$ (there is no control in the differentiation phase) and the maturity speed rate

$$
h_{f}\left(\gamma, u_{f}\right)=\tau_{h f}\left(-\gamma^{2}+\left(c_{1} \gamma+c_{2}\right)\left(1-\omega_{s}(\gamma) e^{-u_{f} / \bar{u}}\right),\right.
$$

where $\omega_{s}(\gamma)$ is nearly constant equal to $1\left(c_{1}, c_{2} \ldots\right.$ are constants given in [14]).

- Mathematical model. The cell population in a follicule $f$ is represented by its cell

${ }^{1}$ sensibility to the fall of plasmatic level of FSH 
density function $\Phi_{f}(t, a, \gamma)$, defined on each cellular phase as solutions of the following conservation laws $[7,30,14,12]$ :

$$
\frac{\partial}{\partial t} \Phi_{f}+\frac{\partial}{\partial a} g_{f} \Phi_{f}+\frac{\partial}{\partial \gamma} h_{f} \Phi_{f}=-\Lambda \Phi_{f}
$$

and the mitosis boundary condition in the G1, S to M phases,

$$
g_{f}(t, a=0, \gamma) \Phi_{f}(t, a=0, \gamma)=2 g_{f}\left(t, a=a_{2}, \gamma\right) \Phi_{f}\left(t, a=a_{2}, \gamma\right),
$$

and in the differentiation phase there is no cell of age 0 ,

$$
\Phi_{f}(t, a=0, \gamma)=0 .
$$

The boundary condition between the G1 and the differentiation phase (through the threshold maturity $\gamma_{s}$ ) is given by :

$$
h_{f}\left(t, a, \gamma_{s}^{+}\right) \Phi_{f}\left(t, a, \gamma_{s}^{+}\right)=h_{f}\left(t, a, \gamma_{s}^{-}\right) \Phi_{f}\left(t, a, \gamma_{s}^{-}\right),
$$

where $w(a-)=\lim _{x \rightarrow a, x<a} w(a)$ and $w(a+)=\lim _{x \rightarrow a, x>a} w(a)$.

- Definition domain. We can summarize the domain definition using the following picture (see fig 1.1)

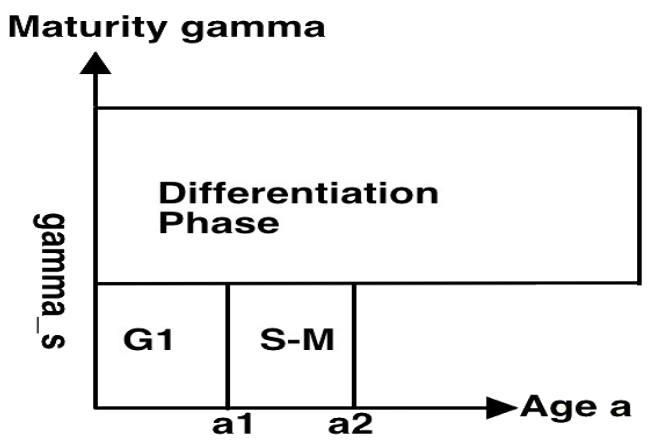

FIG. 1.1. Domain of $G 1, S$ to $M$ and differentiation in age and maturity variables.

This multi-scale model describes the follicle selection process and, here, the assumptions are not motivated by mathematical considerations.

Outline of this work. The aim of this paper is to give a rigorous and full mathematical study of this type of model. The difficulties to analyze this dynamical system of nonlinear coupled partial differential equations proposed by INRIA team Sisyphe in $[14,13]$ are important : we observe that the solutions of these PDEs seems to concentrate their mass around a mean maturity $\gamma$ and the dynamical behavior doesn't seem to be as simple as the the dynamic is in the Lacker's model [21].

Firstly, we give some mathematical results (theorem 2.1) on the multi-scale model which allow us to reduce this model to a simpler one where follicles are defined by 
their mass and their maturity. The mass and the maturity of each follicle follow a dynamical system of coupled nonlinear Ordinary Differential Equations as in the Lacker model [21].

Then, in Section 3, we study this simplified model and we give some results on the sensibility of follicle to the fall of plasmatic level of FSH, i.e. the pressure of all the others follicles on the system. In the theorem 3.11, we show that appears bifurcations and catastrophes (see $[35,1,33,2]$ ) in the dynamic of the follicle evolution.

Finally, in section 4, we give some numerical results and we compare the dynamics of the simpler model to the dynamic of the Lacker model.

2. To a simpler model. In this part, we do not give all the details of the multiscale model [14] such as the definitions of the birth rate, death rate, age speed rate or maturity rate. We only deal with the master equations (1.4)-(1.7) giving the evolution of the cell density of each follicle and the crucial decay property of the maturity speed rate $h_{f}$ with respect to the maturity variable $\gamma$. We notice that $h_{f}$ depend on $\gamma$, the mean maturity $\mathcal{M}_{f}$ and the total maturity $\mathcal{M}_{T}$. We assume that $h_{f}$ is smooth with respect to $\mathcal{M}_{f}$ and $\mathcal{M}_{T}$.

$$
h_{f} \in C^{1},\left.\quad h_{f}\right|_{\gamma=0} \in L^{\infty}, \quad \frac{\partial}{\partial \gamma} h_{f} \leq-\eta<0 .
$$

Under this assumption, we prove that the support of the cell density of each follicle concentrates its mass around a curve given by a characteristic equation. The idea is to drastically simplify the model to make the study easier. We will consider that the follicle $f$ is defined by its mass $\rho_{f}$ which satisfies the equation

$$
\frac{\partial}{\partial t} \rho_{f}=\left[B\left(t, \zeta_{f}(t)\right)-\Lambda\left(t, \zeta_{f}(t)\right)\right] \rho_{f},
$$

where $B$ is a birth rate and $\Lambda\left(t, \zeta_{f}(t)\right)$ is a death rate; and its local maturity $\zeta_{f}$ which follows the evolution equation

$$
\frac{\partial}{\partial t} \zeta_{f}=h_{f}\left(t, \zeta_{f}(t)\right)
$$

2.1. The multi-scale model of follicles evolution. In the multi-scale model of INRIA team Sisyphe [14], at time $t$, the follicle $f$ is characterized by the density $\Phi_{f}(t, a, \gamma)$ of its cells of age $a$ and maturity $\gamma$. The evolution of the follicle cell density depends on which domain the density is supported. More precisely, in the proliferating set $(a, \gamma) \in\left[0, a_{2}\right] \times\left[0, \gamma_{s}[\right.$, the density follows the master equation (1.4). The age speed rate $g_{f}$, the maturity speed rate $h_{f}$ and the mitosis rate $\Lambda$ depend on the whole system of follicles and FSH release. There are three different domains of age and maturity where the dynamics given by the master equations are completely different. In the Proliferation set $\gamma<\gamma_{s}$, the density follows the master equation (1.4) with the mitosis boundary condition (1.5). In the Differentiated set $\gamma>\gamma_{s}$, the density follows the equation (1.4) with the boundary condition $\Phi_{f}(t, a=0, \gamma)=0$ (no mitosis). There exists a cell vulnerability zone in which the apoptosis rate $\Lambda$ is positive. The upper bound of the vulnerability zone $\gamma_{r}$ is the maximum of the support of the apoptosis rate

$$
\gamma_{r}=\max _{\mathcal{M}_{f}, \mathcal{M}_{T}} \operatorname{Supp}\left(\gamma \mapsto \Lambda\left(\mathcal{M}_{f}, \mathcal{M}_{T}, \gamma\right)\right)>\gamma_{s},
$$


then, approximating the local maturity

$$
\gamma \sim \iint \gamma \Phi_{f} d a d \gamma / \iint \Phi_{f} d a d \gamma=\text { Maturity/Mass }
$$

we see that we have three zone : proliferation, vulnerability and differentiated.

2.2. First results on the behavior of the dynamic. This nonlinear model is rather complex, nevertheless we numerically observe that the support of $\Phi_{f}$ has a predictable behavior. More precisely, the support of $\Phi_{f}$ concentrates its mass around $\zeta_{f}(t, a)$ solution to the characteristic equation (see transport equations $[9,10,11,34$, $30,31])$

$$
\frac{\partial}{\partial t} \zeta_{f}+g_{f} \frac{\partial}{\partial a} \zeta_{f}=h_{f}\left(\zeta_{f}, \mathcal{M}_{f}, \mathcal{M}_{T}\right)
$$

with the boundary condition

$$
\zeta_{f}(t, a=0)=\zeta_{f}\left(t, a=a_{2}\right)
$$

Indeed, under the assumptions of the decay of the maturity speed rate $h_{f}(2.1)$ and the localization of the support of $\Phi_{f}(t=0)$,

$$
\operatorname{Supp}\left(\Phi_{f}(t=0)\right) \subset\left[0, a_{2}\right] \times\left[x_{0}, x_{1}\right],
$$

where $0<x_{0}<x_{1}<\gamma_{s}$. We have the following theorem.

Theorem 2.1. Assume (2.1) and (2.7) and let $\zeta_{0}^{s}$ and $\zeta_{1}^{s}$ solutions to (2.5) and (2.6) with the initial data

$$
\zeta_{i}^{s}(0, a)=x_{i}, \quad i=0,1,
$$

where $0<x_{0}<x_{1}<\gamma_{s}$, then, for any positive initial data, a solution $\Phi_{f}$ to the system (1.4)-(1.7), satisfies

$$
\operatorname{Supp}\left(\Phi_{f}(t, . . .)\right) \subset\left\{\{a\} \times\left[\zeta_{0}^{s}(t, a), \zeta_{1}^{s}(t, a)\right], \quad a \in\left[0, a_{2}\right]\right\}, \quad \forall t \geq 0,
$$

with the concentration of the support

$$
\sup _{a}\left|\zeta_{0}^{s}(t, a)-\zeta_{1}^{s}(t, a)\right|^{2} \leq C s t e^{-2 \eta t},
$$

where $\eta$ is defined in (2.1).

Moreover, there exists a singular weak solutions to (1.4)-(1.7),

$$
\tilde{\Phi}(t, a, \gamma)=\rho_{f}(t, a) \delta_{\gamma=\zeta_{f}(t, a)},
$$

with $\rho_{f}$ solution to the proliferation McKendrick-VonFoerster equations

$$
\begin{gathered}
\frac{\partial}{\partial t} \rho_{f}+\frac{\partial}{\partial a} g_{f} \rho_{f}=-\Lambda \rho_{f}, \\
g_{f}(t, a=0, \gamma) \rho_{f}(t, a=0, \gamma)=2 g_{f}\left(t, a=a_{2}, \gamma\right) \rho_{f}\left(t, a=a_{2}, \gamma\right),
\end{gathered}
$$


and the initial condition,

$$
\zeta_{f}(0, a) \in\left\{f \in C ^ { 0 } \left(\left[0, a_{2}\left[, f(0)=f\left(a_{2}\right)\right\}, \quad \rho_{f}(0, a) \in L^{1}\left(\left[0, a_{2}\right]\right),\right.\right.\right.
$$

where the mass $\iint_{\mathcal{S}} \Phi_{f} d a d \gamma$ is equal to $\int_{0}^{a_{2}} \rho_{f} d a$ and the maturity $\mathcal{M}_{f}=\int_{0}^{\infty} \zeta_{f} \rho_{f} d a$.

Proposition 2.2. Let $\zeta_{f}$ solution to (2.5) and (2.6) and $\rho_{f}$ solution to (2.12) and (2.13) then $\tilde{\Phi}(t, a, \gamma)$ defined by (2.11) is a weak solution to (1.4)-(1.7).

Therefore, the singular case (weak solution given by the proposition 2.2) is the limit case of any solution to the main system of evolution equations (1.4)-(1.7).

2.3. Proof of Theorem 2.1. We can easily see there exists $\Psi_{f}>0$ solution to the dual (backward) equation

$$
\frac{\partial}{\partial t} \Psi_{f}+g_{f} \frac{\partial}{\partial a} \Psi_{f}+h_{f} \frac{\partial}{\partial \gamma} \Psi_{f}=\Lambda \Psi_{f}
$$

with the boundary conditions

$$
\begin{gathered}
\Psi_{f}(t, a=0, \gamma)=\frac{1}{2} \Psi_{f}\left(t, a=a_{2}, \gamma\right), \\
\Psi_{f}(t, a, \gamma=0)=1+a / 2,
\end{gathered}
$$

and the initial data

$$
\Psi_{f}(t, a, \gamma)=1+a / 2
$$

Indeed, $\Psi_{f}$ follows a transport equation (2.15) and using the characteristics, we have the existence and uniqueness of the solution (see $[30,27]$ ). Then, we have directly the following lemma.

Lemma 2.3. Let $F \in C^{1}((-\infty, \infty),(-\infty, \infty)),(\zeta, \Psi)$ solution to (2.5), (2.6), (2.15) and (2.16) then we have the conservation law

$$
\iint_{\mathcal{S}} \Phi_{f}(t, a, \gamma) \Psi_{f}(t, a, \gamma) d a d \gamma=C s t
$$

and

$$
\begin{aligned}
& \frac{d}{d t} \iint_{\mathcal{S}} \Phi_{f}(t, a, \gamma) \Psi_{f}(t, a, \gamma) F\left(\gamma-\zeta_{f}(t, a)\right) d a d \gamma \\
& \quad=\iint_{\mathcal{S}} \Phi_{f}(t, a, \gamma) \Psi_{f}(t, a, \gamma) F^{\prime}\left(\gamma-\zeta_{f}(t, a)\right)\left[h_{f}(\gamma)-h_{f}\left(\zeta_{f}\right)\right] d a d \gamma
\end{aligned}
$$

Proof. The first conservation law comes from the duality between $\Phi_{f}$ and $\Psi_{f}$ (see $[26,30,31,32,25])$ since

$$
\frac{\partial}{\partial t} \Psi_{f} \Phi_{f}+\frac{\partial}{\partial a} g_{f} \Psi_{f} \Phi_{f}+\frac{\partial}{\partial \gamma} h_{f} \Psi_{f} \Phi_{f}=0
$$

and $g_{f} \Psi_{f} \Phi_{f}(a=0)=g_{f} \Psi_{f} \Phi_{f}\left(a=a_{2}\right)$ in the proliferation zone (resp. $g_{f} \Psi_{f} \Phi_{f}(a=$ $0)=g_{f} \Psi_{f} \Phi_{f}(a=\infty)=0$ in the differentiated zone.) Then, using (2.5) we have that

$$
\begin{aligned}
\frac{\partial}{\partial t}\left(\Psi_{f} \Phi_{f} F\right. & \left.\left(\gamma-\zeta_{f}(t, a)\right)\right)+\frac{\partial}{\partial a}\left(g_{f} \Psi_{f} \Phi_{f} F\left(\gamma-\zeta_{f}(t, a)\right)\right) \\
& \left.+\frac{\partial}{\partial \gamma}\left(h_{f} \Psi_{f} \Phi_{f} F\left(\gamma-\zeta_{f}(t, a)\right)\right)\right)=F^{\prime}\left(\gamma-\zeta_{f}(t, a)\right)\left[h_{f}(\gamma)-h_{f}(\zeta)\right] \Psi_{f} \Phi_{f}
\end{aligned}
$$


and (2.20) follows.

Now, assuming that $h_{f}$ decreases with respect to $\gamma$, we find that $\Phi_{f} \Psi_{f}$ concentrates its mass around $\zeta_{f}$. More precisely, using lemma 2.3 with $F(x)=x^{2}$, $F(x)=(x)_{+}^{2}=(\max (x, 0))^{2}$ and $F(x)=(x)_{-}^{2}=(\min (x, 0))^{2}$ we have directly the following lemma.

LEMMA 2.4. Under assumption (2.1), we have that

$$
d \nu(t, a, \gamma)=\Phi_{f}(t, a, \gamma) \Psi_{f}(t, a, \gamma) d a d \gamma
$$

is a probability measure which satisfies

$$
\begin{aligned}
& \frac{d}{d t} \iint_{\mathcal{S}}\left(\gamma-\zeta_{f}(t, a)\right)^{2} d \nu \leq-2 \eta \iint_{\mathcal{S}}\left(\gamma-\zeta_{f}(t, a)\right)^{2} d \nu \\
& \frac{d}{d t} \iint_{\mathcal{S}}\left(\gamma-\zeta_{f}(t, a)\right)_{+}^{2} d \nu \leq-2 \eta \iint_{\mathcal{S}}\left(\gamma-\zeta_{f}(t, a)\right)_{+}^{2} d \nu,
\end{aligned}
$$

and

$$
\frac{d}{d t} \iint_{\mathcal{S}}\left(\gamma-\zeta_{f}(t, a)\right)_{-}^{2} d \nu \leq-2 \eta \iint_{\mathcal{S}}\left(\gamma-\zeta_{f}(t, a)\right)_{-}^{2} d \nu
$$

We finally conclude the proof of the theorem 2.1 using that the support of $\Phi_{f}$ belongs to $\left[0, a_{2}\right] \times\left[x_{0}, x_{1}\right]$ (assumption (2.7)). We let $\zeta_{0}^{s}, \zeta_{1}^{s}$ solutions to (2.5) and (2.6) with the initial data (2.8). Then, it suffices to use lemma 2.4 and notice that $\Psi_{f}(t, a, \gamma)>0$ for all $t>0$, age $a$ and maturity $\gamma$ as soon as $\Psi_{f}(0, a, \gamma)>0$. Indeed, we have then $\operatorname{Supp} \Phi_{f}=\operatorname{Supp} \Phi_{f} \Psi_{f}$ and we can conclude using that

$$
\begin{aligned}
& \iint_{\mathcal{S}}\left(\gamma-\zeta_{1}^{s}(0, a)\right)_{+}^{2} d \nu(0, a, \gamma)=0, \\
& \iint_{\mathcal{S}}\left(\gamma-\zeta_{0}^{s}(0, a)\right)_{-}^{2} d \nu(0, a, \gamma)=0,
\end{aligned}
$$

and (2.22)-(2.23). The result of convergence (2.13) comes from the inequality

$$
\frac{\partial}{\partial t}\left(\zeta_{0}^{s}-\zeta_{1}^{s}\right)^{2}+g_{f} \frac{\partial}{\partial a}\left(\zeta_{0}^{s}-\zeta_{1}^{s}\right)^{2}=2\left(\zeta_{0}^{s}-\zeta_{1}^{s}\right)^{2} \frac{h_{f}\left(\zeta_{0}^{s}\right)-h_{f}\left(\zeta_{1}^{s}\right)}{\zeta_{0}^{s}-\zeta_{1}^{s}} \leq-2 \eta\left(\zeta_{0}^{s}-\zeta_{1}^{s}\right)^{2}
$$

with the boundary condition $\left(\zeta_{0}^{s}-\zeta_{1}^{s}\right)^{2}(a=0)=\left(\zeta_{0}^{s}-\zeta_{1}^{s}\right)^{2}\left(a=a_{2}\right)$.

2.4. Proof of Proposition 2.2. We recall that $\Phi_{f}$ is a weak solution to (1.4)(1.7), if, for all $\chi \in C_{0}^{\infty}\left(\left[0, \infty\left[^{2}\right)\right.\right.$, we have (in distributional sense)

$$
\begin{aligned}
& \frac{\partial}{\partial t}\left(\Phi_{f}, \chi\right)-\left(g_{f} \Phi_{f}, \frac{\partial}{\partial a} \chi\right)-\left(h_{f} \Phi_{f}, \frac{\partial}{\partial \gamma} \chi\right) \\
& \quad=\left[\left(g_{f} \Phi_{f}(a=0), \chi(a=0)\right)-\left(g_{f} \Phi_{f}\left(a=a_{2}\right), \chi\left(a=a_{2}\right)\right)\right]-\left(\Lambda \Phi_{f}, \chi\right) .
\end{aligned}
$$

We now prove that $\tilde{\Phi}=\delta_{\gamma=\zeta} \rho_{f}$ is a weak solution. We find that

$$
\left(g_{f} \Phi_{f}, \frac{\partial}{\partial a} \chi\right)=\int \rho_{f} g_{f}\left(\frac{\partial}{\partial a} \chi\right)\left(a, \zeta_{f}(t, a)\right) d a,
$$




$$
\begin{gathered}
\left(h_{f} \Phi_{f}, \frac{\partial}{\partial \gamma} \chi\right)=\int \rho_{f} h_{f} \frac{\partial}{\partial \gamma} \chi\left(a, \zeta_{f}(t, a)\right) d a \\
\left(\Lambda \Phi_{f}, \chi\right)=\int \Lambda\left(t, a, \zeta_{f}(t, a)\right) \rho_{f} \chi\left(a, \zeta_{f}(t, a)\right) d a \\
\left(\Phi_{f}, \chi\right)=\int \rho_{f}(t, a) \chi\left(a, \zeta_{f}(t, a)\right) d a
\end{gathered}
$$

thus, we find that

$$
\frac{\partial}{\partial t}\left(\Phi_{f}, \chi\right)=\int \chi\left(a, \zeta_{f}(t, a)\right) \frac{\partial}{\partial t} \rho_{f}(t, a) d a+\int \rho_{f}(t, a) \frac{\partial}{\partial t} \zeta_{f}(t, a)\left(\frac{\partial}{\partial \gamma} \chi\right)\left(a, \zeta_{f}(t, a)\right) d a .
$$

Then, using (2.12), (2.13), (2.5) and (2.6) we have directly that (2.24) is satisfied for $\tilde{\Phi}$.

We notice that $d \nu$ is a probability measure, and the inequality (2.21) means that the Wasserstein distance, see [29], between $d \nu$ and $\delta_{\gamma=\zeta_{f}(t, a)}$ vanishes as $t \rightarrow \infty$ and so the measure $d \nu$ concentrates its mass around $\zeta_{f}(t, a)$ (see for analogous problems $[25,9,19,20])$.

2.5. Conclusion and reduction of the model. The singular case in which the cell density is a dirac mass with respect to the maturity variable $\gamma$ is biologically meaningful. This corresponds to the case where all the cells of the follicle have the same maturity. Theorem 2.1 show that, in the long time behavior, for each follicle, cells of the follicle get organized and make uniform their maturity. Thus, we can reduce the model to the evolution of the mass equation (proliferation equation)

$$
\begin{gathered}
\frac{\partial}{\partial t} \rho_{f}+\frac{\partial}{\partial a} g_{f} \rho_{f}=-\Lambda \rho_{f}, \\
g_{f}(t, a=0, \gamma) \rho_{f}(t, a=0, \gamma)=2 g_{f}\left(t, a=a_{2}, \gamma\right) \rho_{f}\left(t, a=a_{2}, \gamma\right),
\end{gathered}
$$

and the maturity equation

$$
\frac{\partial}{\partial t} \zeta_{f}+g_{f} \frac{\partial}{\partial a} \zeta_{f}=h_{f}
$$

with the boundary condition

$$
\zeta_{f}(t, a=0, \gamma)=\zeta_{f}(t, a=2, \gamma),
$$

where the mass $\iint \Phi_{f} d a d \gamma$ is equal to $\int \rho_{f} d a$ and the maturity $\mathcal{M}_{f}=\int \zeta_{f} \rho_{f} d a$. We notice that, in the Differentiated domain, we have $g_{f}(t, a=0, \gamma) \rho_{f}(t, a=0, \gamma)=0$, thus integrating (2.25) with respect to $a$, we find that

$$
\frac{\partial}{\partial t} \int \rho_{f} d a=-\int \Lambda \rho_{f} d a .
$$

Moreover, we see in [14] that $h_{f}$ does not depend on $a$ in the Differentiated domain, thus $\zeta_{f}(t, a)=\zeta_{f}(t)$ is solution to

$$
\frac{\partial}{\partial t} \zeta_{f}=h_{f}
$$


and $\Lambda\left(t, \zeta_{f}(t, a)\right)=\Lambda\left(t, \zeta_{f}(t)\right)$ implying that $\frac{\partial}{\partial t} \int \rho_{f} d a=-\Lambda\left(t, \zeta_{f}(t)\right) \int \rho_{f} d a$. Therefore, we see that the death rate of (2.2) is given by $\Lambda\left(t, \zeta_{f}(t)\right)$ and we notice that (2.2) and (2.3) are solutions to (2.29) and (2.30).

The simplifications we have to do are in the Proliferation domain, here we choose the birth rate of $(2.2)$ as

$$
B(t)=g\left(t, \zeta_{f}(t)\right) \ln (2) / a_{2} .
$$

Indeed, during the proliferation process, the number of cells is multiplied by 2 (term $\ln (2))$ after a time $T \sim \frac{\text { age speed rate }}{\text { age } \max }\left(\operatorname{term} g\left(t, \zeta_{f}(t)\right) / a_{2}\right)$. Nevertheless, this simplification hides a delay which appears in the McKendrick VonFoerster type equations of evolution (2.26) (see [7, 37, 17, 18, 30]).

Remark. We notice that, in the model [14], the maturity speed rate vanishes in a sub domain of the proliferation zone, but the method we use to prove the convergence to a dirac mass holds.

3. Follicles evolution : a mass and maturity dynamical system. We summarize the simplified model as a system of ODE (as in the Lacker model [21]) where the maturity follows the evolution equation

$$
\frac{\partial}{\partial t} \zeta_{f}=h_{f}\left(t, \zeta_{f}(t)\right)
$$

and where the mass of each follicle satisfies an ODE

$$
\frac{\partial}{\partial t} \rho_{f}=\left[B\left(t, \zeta_{f}(t)\right)-\Lambda\left(t, \zeta_{f}(t)\right)\right] \rho_{f}=\Delta\left(t, \zeta_{f}(t)\right) \rho_{f},
$$

where $B$ is the birth rate

$$
B(t)=g\left(t, \zeta_{f}(t)\right) \ln (2) / a_{2},
$$

$\Lambda\left(t, \zeta_{f}(t)\right)$ is a death rate and $\Delta$ the global growth rate. In order to study more precisely the behavior of $\left(\rho_{f}, \zeta_{f}\right)$, we have to introduce the speed rate functions and the death rate function.

- System of Ordinary Differential Equations. Since the measurable maturity is the mean maturity $\mathcal{M}_{f}=\rho_{f} \zeta_{f}$ and not $\zeta_{f}$, it is more interesting to study the evolution system

$$
\left\{\begin{array}{l}
\frac{\partial}{\partial t} \mathcal{M}_{f}=\rho_{f} h_{f}\left(\mathcal{M}_{f}, \mathcal{M}_{T}, \mathcal{M}_{f} / \rho_{f}\right)+\Delta\left(\mathcal{M}_{f}, \mathcal{M}_{T}, \mathcal{M}_{f} / \rho_{f}\right) \mathcal{M}_{f} \\
\frac{\partial}{\partial t} \rho_{f}=\Delta\left(\mathcal{M}_{f}, \mathcal{M}_{T}, \mathcal{M}_{f} / \rho_{f}\right) \rho_{f}
\end{array}\right.
$$

Therefore, the follicle $f$ is defined by its mass and its mean maturity $\left(\rho_{f}, \mathcal{M}_{f}\right)$ which follows the master system of equations (3.1). 
- Domains definition. There are also three zone, the zone where the birth rate $B>0$ is strictly positive ("proliferation"),

$$
\Pi_{\text {Proliferation }}=\left\{\left(\text { maturity } \mathcal{M}_{f}, \text { mass } \rho_{f}\right): \text { maturity } / \text { mass }<\gamma_{s}\right\} \text {, }
$$

the zone of differentiated follicles where the birth rate vanishes $(B=0)$

$$
\Pi_{\text {Differentiated }}=\left\{(\text { maturity }, \text { mass }): \text { maturity } / \text { mass } \geq \gamma_{s}\right\},
$$
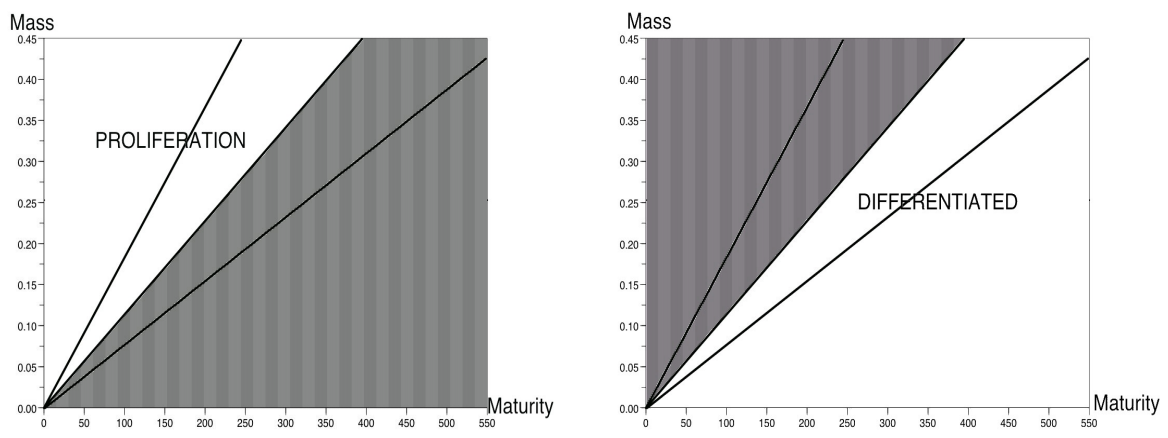

FIG. 3.1. Proliferation and Differentiated zone $(\Lambda>0)$

the vulnerable, or sensitive, zone where the apoptosis rate is strictly positive

$$
\Pi_{\text {Vulnerable }}=\left\{(\text { maturity, mass }): \gamma_{t} \leq \text { maturity } / \text { mass } \leq \gamma_{r}\right\},
$$

where $\gamma_{r}<\gamma_{s}<\gamma_{t}$ (corresponding to the support of the apoptosis rate) and for convenience, we define a saved zone where the apoptosis rate vanishes $(\Lambda=0)$

$$
\Pi_{\text {Saved }}=\left\{(\text { maturity, mass }): \text { maturity } / \text { mass }>\gamma_{r}\right\} .
$$
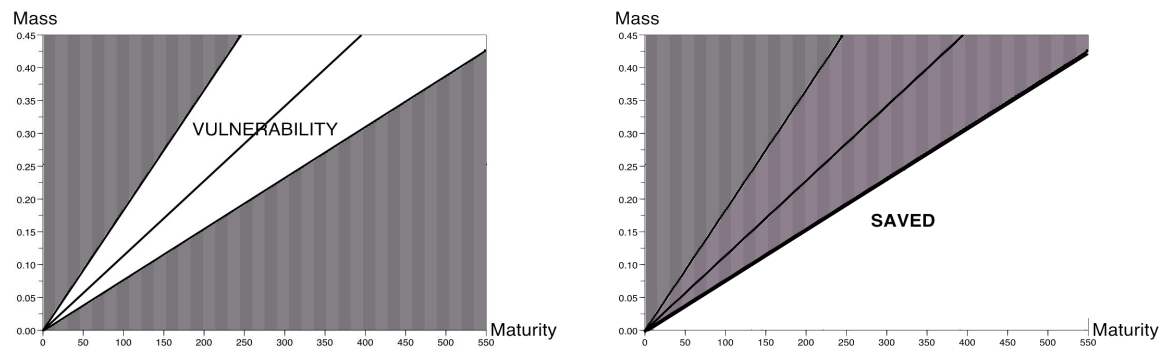

FIG. 3.2. sensitive and Saved zone

We notice here that, without any other assumptions on the birth rate $B$, apoptosis rate $\Lambda$ and on the maturity speed rate $h_{f}$, steady states can appear in every zone 
except in the domain $\left\{(\right.$ maturity, mass $):$ maturity $>0 \quad \gamma_{s} \leq$ maturity/mass $\leq$ $\left.\gamma_{r}\right\}$. Nevertheless, we directly see (eq. (3.1)) that a steady states must satisfy $h_{f}\left(\mathcal{M}_{f}, \mathcal{M}_{T}, \mathcal{M}_{F} / \rho_{f}\right)=0$ for all $f$, so we have to study more carefully the zeros of $h_{f}$. Since the maturity speed rate $h_{f}$ depends on $\mathcal{M}_{T}$, change the value of the maturity of only one follicle in a system of follicles at equilibrium makes all the system be out of equilibrium. More precisely we see that the evolution of the maturity $\mathcal{M}_{f}$ of the follicle $f$ depends on the maturity $\mathcal{M}=\mathcal{M}_{T}-\mathcal{M}_{f}$ of all others follicles. This maturity $\mathcal{M}$ can be considered as an exogenous "parameter" for the follicle $f$ and then changing its value makes the follicle $f$ die or survive. We'll see that appear bifurcations and catastrophes $[8,16,33,35]$ in the dynamical system (3.1).

In section 3.1, we give some definitions to make the study of the dynamical system (3.1) easier then in section 3.2, we give the main result on the behavior of this system. Finally, in section 3.3, we prove the main theorem.

3.1. Definitions. In order to study the dynamic of the system (3.1) which hardly depends on $h_{f}$, we have to search the possible steady states of the system and the geometry of the steady states set. Moreover, we have to see the variation of the steady state of a follicle when the others have their mass and maturity which change.

3.1.1. Steady states. We have to define two functions $\zeta_{0}$ and $\rho_{0}$. From now, we use the maturity $\mathcal{M}_{f}$ and the maturity of all the others follicles $\mathcal{M}$. We recall that $h_{f}$ is a function of $\gamma, \mathcal{M}_{f}$ and the total maturity $\mathcal{M}_{T}$, to avoid multiple definition we write again $h_{f}$ as a function of $\gamma, \mathcal{M}_{f}, \mathcal{M}: h_{f}\left(\mathcal{M}_{f}, \mathcal{M}, \gamma\right)$.

Definition 3.1. Since $h$ is strictly decreasing (2.1), we can define $\zeta_{0}$ as the zero of $h_{f}$,

$$
h_{f}\left(\mathcal{M}_{f}, \mathcal{M}, \zeta_{0}\left(\mathcal{M}_{f}, \mathcal{M}\right)\right)=0
$$

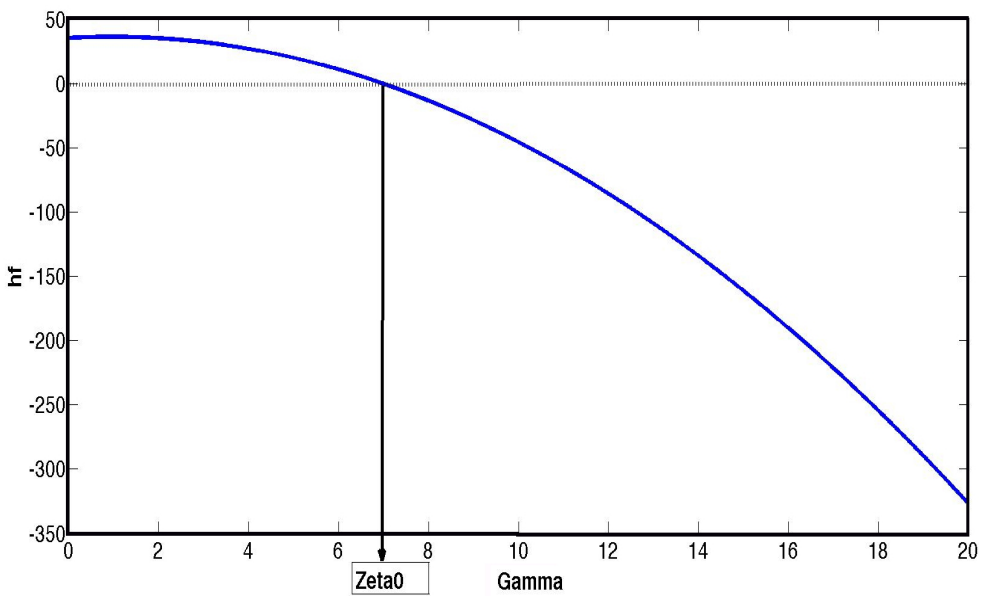

FIG. 3.3. fixing $\left(\mathcal{M}_{f}, \mathcal{M}\right)$ we compute $h_{f}$ and we find the root $\zeta_{0}$ 
Definition 3.2. We define $\rho_{0}$ as,

$$
\rho_{0}\left(\mathcal{M}_{f}, \mathcal{M}\right)=\mathcal{M}_{f} / \zeta_{0}\left(\mathcal{M}_{f}, \mathcal{M}\right),
$$

the graph of $\rho_{0}$ for a maturity of all the others follicles $\mathcal{M}$ as,

$$
\Gamma_{\rho_{0}}(\mathcal{M})=\left\{\left(m, \rho_{0}(m, \mathcal{M})\right), \quad m \geq 0\right\}
$$

and finally

$$
\Omega_{\text {lim }}:=\bigcup_{\mathcal{M} \geq 0} \Gamma_{\rho_{0}}(\mathcal{M})
$$
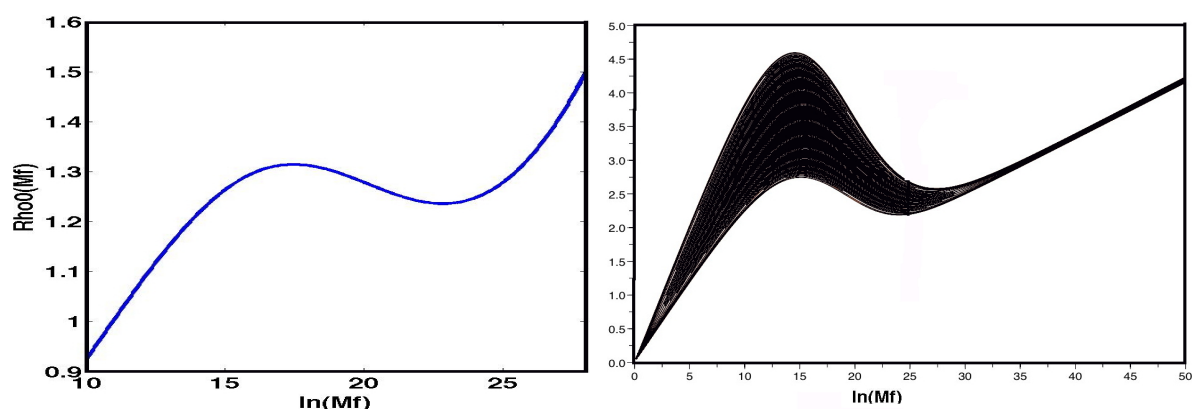

FIG. 3.4. fixing $\mathcal{M}$ we compute $\mathcal{M}_{f} \rightarrow \rho_{0}\left(\mathcal{M}_{f}, \mathcal{M}\right)$ and $\Omega_{\text {lim }}$

We notice here, that, the set of steady states of (3.1) belongs to $\Omega_{\text {lim }}$. Moreover, for a given maturity $\mathcal{M}$, the set of steady states of (3.1) belongs to $\left[0, \infty\left[\times\left\{\rho_{f}^{E}\right\} \cap \Gamma_{\rho_{0}}(\mathcal{M})\right.\right.$ where $\rho_{f}^{E}$ is the mass of the follicle as it enters in the saved zone $\Pi_{\text {saved }}$.

DEFINITION 3.3. We define $\rho_{f}^{E}$ as the value of $\rho_{f}$, at the first time the follicle $f$ enters in the saved zone

$$
\rho_{f}^{E}=\rho_{f}\left(\inf \left\{t:\left(\mathcal{M}_{f}, \rho_{f}\right)(t) \in \Pi_{\text {Saved }}\right\}\right),
$$

with $\rho_{f}^{E}=0$ as $\inf \left\{t:\left(\mathcal{M}_{f}, \rho_{f}\right)(t) \in \Pi_{\text {Saved }}\right\}=\infty$.

In order to have some generic assumptions, we define the set $\Omega_{c}$, which gives, when $\mathcal{M}$ is fixed, where the function $\mathcal{M}_{f} \rightarrow \rho_{0}\left(\mathcal{M}_{f}, \mathcal{M}\right)$ is decreasing (and ${ }^{c} \Omega_{c}$ where it increases)).

Definition 3.4. We define $\Omega_{c}(\mathcal{M})$,

$$
\Omega_{c}(\mathcal{M})=\left\{m: m \frac{\partial}{\partial m} \log \left(\zeta_{0}(m, \mathcal{M})\right) \geq 1\right\}=\left\{m: \frac{\partial}{\partial m} \rho_{0}(m, \mathcal{M}) \leq 0\right\}
$$

Definition 3.5. We define $N_{c c}(\mathcal{M})$ as the number of connex component of $\Omega_{c}(\mathcal{M})$ and

$$
C_{p}=\max _{\mathcal{M}} N_{c c}(\mathcal{M})
$$

The number $C_{p}$ gives the number of times $\frac{\partial}{\partial m} \rho_{0}(m, \mathcal{M})$ changes sign, i.e., $\mathcal{M}_{f} \rightarrow$ $\rho_{0}\left(\mathcal{M}_{f}, \mathcal{M}\right)$ changes from decay to growth. 
3.1.2. Bifurcations and catastrophes. Here, we need to study the geometry of $\mathcal{D}\left(\rho_{f}^{E}\right) \bigcap \Gamma_{\rho_{0}}(\mathcal{M})$ and $\mathcal{D}\left(\rho_{f}^{E}\right) \bigcap \Gamma_{\rho_{0}}(\mathcal{M}) \bigcap \Pi_{\text {saved }}$, where $\mathcal{D}\left(\rho_{f}^{E}\right)=\left[0, \infty\left[\times\left\{\rho_{f}^{E}\right\}\right.\right.$, when the exogenous maturity $\mathcal{M}$ changes. Therefore, we can consider that the other follicles induce a pressure on the follicle $f$ through their maturity. We introduce a function which measure how sensitive is a follicle to the pressure of the other follicles. Then we define three cases :

- the follicle is not sensitive to the pressure of the other follicles and so we consider that its a follicle which will survive and will participate to the reproduction process,

- the follicle can possibly return (or stay) in the vulnerable zone (where there is no other steady state than 0 ) when the pressure of the other follicle is sufficient (this follicle is doomed by the pressure of the other follicles),

- the follicle can possibly return in the vulnerable zone or reach a "less sensitive to the pressure" zone and the dynamic of the follicle strongly depends on the dynamic of the other follicle (we say that catastrophes can happen).

Definition 3.6. We define $N\left(\rho_{f}^{E} \mid \mathcal{M}\right)$ as the number of connex component of the intersection of $\Gamma_{\rho_{0}}(\mathcal{M})$ with the horizontal line (see fig. 3.5, 3.6)

$(3.13) N\left(\rho_{f}^{E} \mid \mathcal{M}\right):=$ Number of Connex Comp. of $\mathcal{D}\left(\rho_{f}^{E}\right) \bigcap \Gamma_{\rho_{0}}(\mathcal{M}) \bigcap \Pi_{\text {saved }}$.
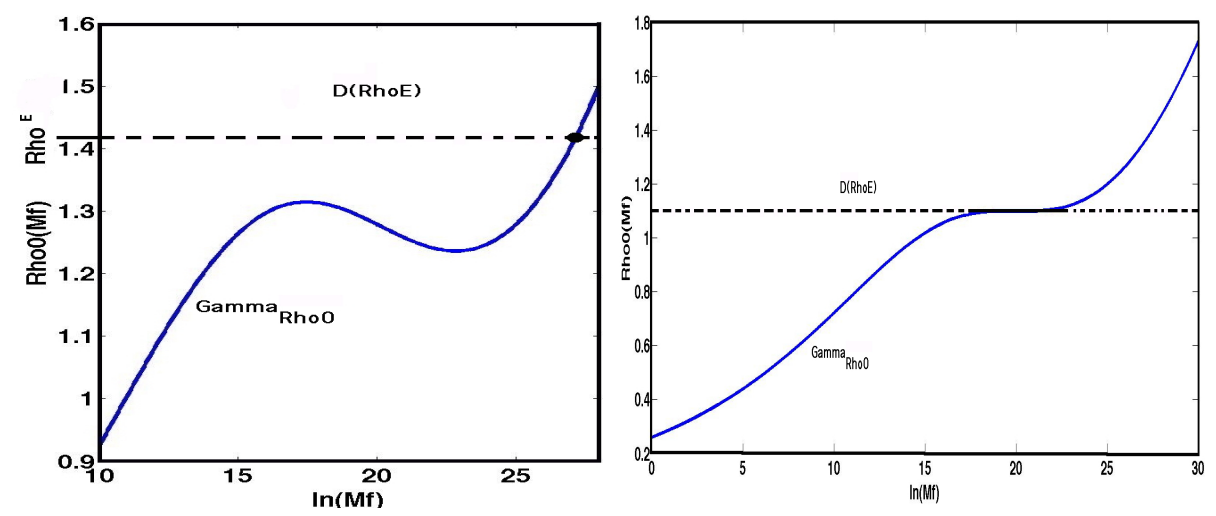

FIG. 3.5. Here we have the case $N\left(\rho_{f}^{E} \mid \mathcal{M}\right)=1$

DEFINITION 3.7. We say that a follicle is insensitive to the pressure if

$$
\mathcal{M} \mapsto N\left(\rho_{f}^{E} \mid \mathcal{M}\right) \text { is constant. }
$$

we say that a follicle is doomed by the pressure if

$$
\exists \mathcal{M}_{*} \text {, s.t. } N\left(\rho_{f}^{E} \mid \mathcal{M}\right)=0, \quad \forall \mathcal{M}>\mathcal{M}_{*},
$$



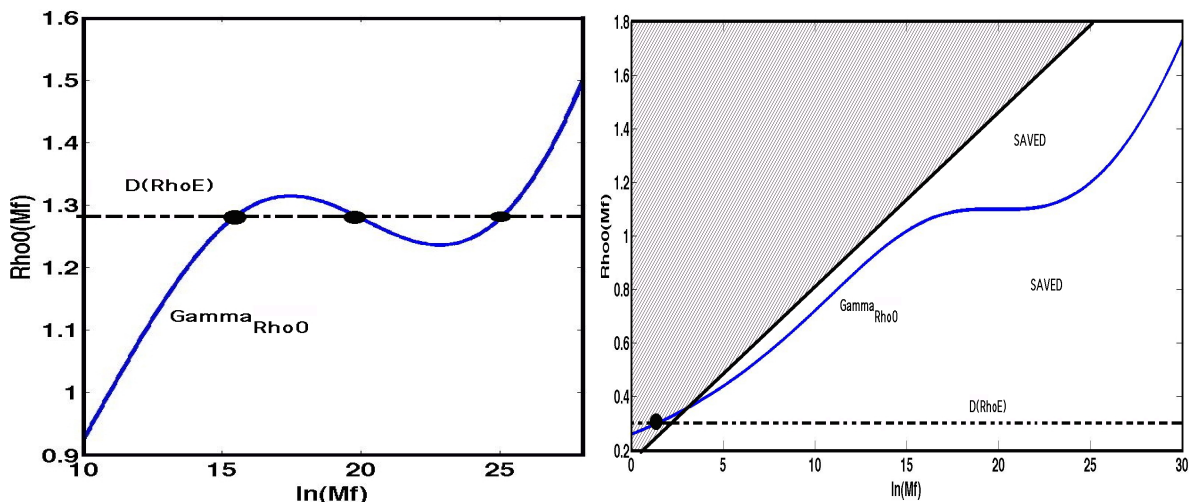

FIG. 3.6. Here we have the case $N\left(\rho_{f}^{E} \mid \mathcal{M}\right)=3$ (left) and when $N\left(\rho_{f}^{E} \mid \mathcal{M}\right)=0$ the intersection is empty in the saved zone

we say there is a catastrophe if

$$
\mathcal{M} \mapsto N\left(\rho_{f}^{E} \mid \mathcal{M}\right) \text { is not constant. }
$$

Example In this example (figure 3.7), we have in the black part : the proliferation zone and sensitive zone. We plot $\Gamma_{\rho_{0}}(\mathcal{M})$ for $\mathcal{M}=0$ to $\mathcal{M}=\infty$. We see that, in the insensitive zone, $\mathcal{D}\left(\rho_{f}^{E}\right) \cap \Gamma_{\rho_{0}}(\mathcal{M}) \bigcap \Pi_{\text {saved }}$ is a point and $N\left(\rho_{f}^{E} \mid \mathcal{M}\right)=1$ for all $\mathcal{M}$. We have that, in the doomed zone, for $\mathcal{M}$ large enough $\mathcal{D}\left(\rho_{f}^{E}\right) \bigcap \Gamma_{\rho_{0}}(\mathcal{M}) \bigcap \Pi_{\text {saved }}$ is empty and $N\left(\rho_{f}^{E} \mid \mathcal{M}\right)=0$.
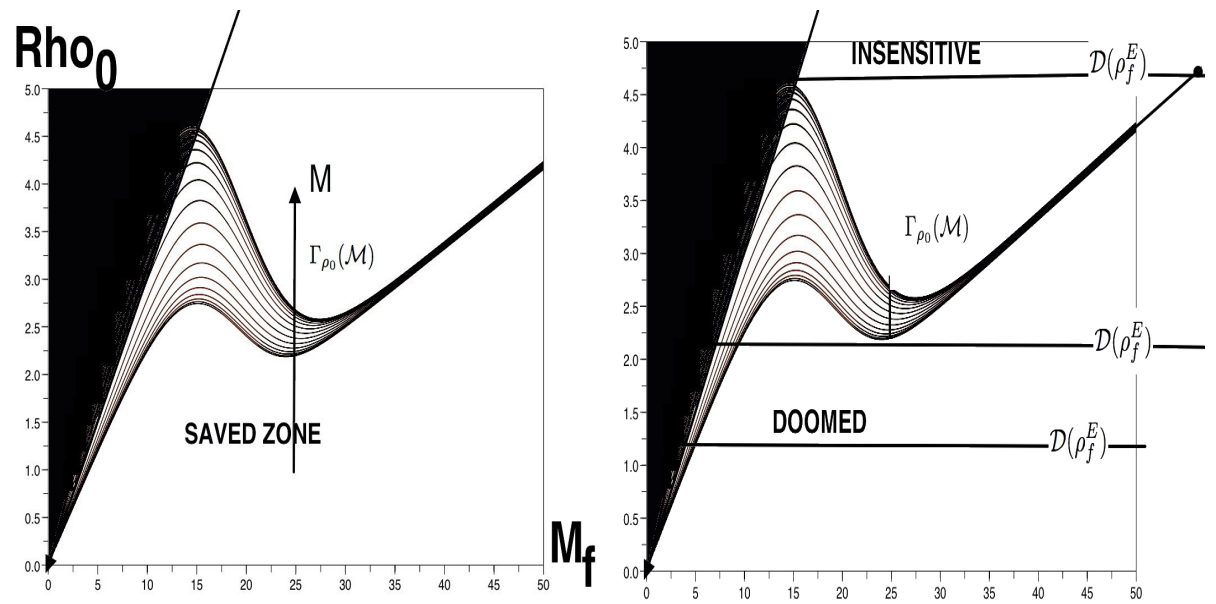

FIG. 3.7. Left : $\mathcal{D}\left(\rho_{f}^{E}\right) \bigcap \Gamma_{0}$. Right : We give three straight lines $\mathcal{D}\left(\rho_{f}^{E}\right)$, the above one intersect $\Gamma_{0}$ (case insensitive $N\left(\rho_{f}^{E} \mid \mathcal{M}\right)=1$ ). The two below are doomed, i.e., for $\mathcal{M}$ large enough, $\mathcal{D}\left(\rho_{f}^{E}\right) \bigcap \Gamma_{\rho_{0}}(\mathcal{M}) \bigcap \Pi_{\text {saved }}$ is empty $\left(N\left(\rho_{f}^{E} \mid \mathcal{M}\right)=0\right)$. 
3.2. Main result on the dynamic of the simpler model. In this part, we give the main biological observations on the follicles growth and selection process. Then, we translate these observations into mathematical assumptions (I-IV). Finally, we give the main theorem on the behavior of follicles in the growth and selection process (3.1). We observe, biologically, that

Observation $I$ : At the end of the selection process there is no follicle in the proliferating zone, i.e., $\gamma>\gamma_{s}$ and the maturity is upper bounded, i.e., $\gamma<\gamma_{\max }$.

Observation II : More the pressure exerted by the follicles on the system is high, less a follicle is able to survive.

Observation III : Large mature follicles have, nearly, the same ratio Maturity/Mass.

Now, we give some hypothesis on the maturity speed rate to be sure that the observations are satisfied by the simplified model (3.1).

ASSUMPTION I. Without any information on the birth rate and the death rate, we have to assume that

$$
0<\gamma_{s}<\inf _{\mathcal{M}_{f}, \mathcal{M}} \zeta_{0}\left(\mathcal{M}_{f}, \mathcal{M}\right) \leq \sup _{\mathcal{M}_{f}, \mathcal{M}} \zeta_{0}\left(\mathcal{M}_{f}, \mathcal{M}\right) \leq \gamma_{\max }<\infty
$$

We notice that this condition is satisfied for a large class of function $h_{f}$, indeed we have the following lemma.

Lemma 3.8. There exists $0<\gamma_{s}<\gamma_{\max }<\infty$ so that condition (3.17) is verified when (2.1) is satisfied and there exists $\underline{x}>0$ so that $\inf _{\mathcal{M}_{f}, \mathcal{M}} h_{f}\left(\mathcal{M}_{f}, \mathcal{M}, x\right)>0$ for all $x<\underline{x}$.

ASSUMPTION II. We assume that, for a given follicle, the maturity speed rate is decreasing with respect to the mass of all others follicles

$$
\mathcal{M} \mapsto h_{f}\left(\mathcal{M}_{f}, \mathcal{M}, \mathcal{M}_{f} / \rho_{f}\right) \text { is strictly decreasing. }
$$

ASSUMPTION III. Large mature follicles have, nearly, the same ratio Maturity/Mass

$$
\zeta_{0}(m, \mathcal{M})=\zeta_{0}^{\infty}+o(1 / m) .
$$

Lemma 3.9. Condition (3.19) is satisfied when $h_{f}$ verifies

$$
\frac{\partial}{\partial \mathcal{M}_{f}} h_{f} \in L^{1}\left(\mathcal { M } _ { f } \in \left[0, \infty\left[, L^{\infty}\left(\mathcal{M} \in\left[0, \infty\left[, \gamma \in\left[\inf _{M, m} \zeta_{0}(M, m) \leq \sup _{M, m} \zeta_{0}(M, m)\right]\right)\right) .\right.\right.\right.\right.
$$

ASSUMPTION IV. To simplify our study of the dynamical system, we restrict ourselves to simple (but not to simple) dynamics of the selection process of follicles (3.1) : we assume that the complexity number $C_{p}$ less than 1

$$
C_{p} \leq 1
$$

LEMma 3.10. The hypothesis (3.20) is verified when $h_{f}$ satisfies 


$$
\begin{aligned}
\forall \mathcal{M}, \quad \exists \mathcal{M}^{*}: \mathcal{M}_{f} \mapsto \mathcal{M}_{f} \frac{\partial}{\partial \mathcal{M}_{f}} \log \left(\zeta_{0}\right)\left(\mathcal{M}_{f}, \mathcal{M}\right), \\
\quad \text { strictly increases on }\left[0, M^{*}[\text { and strictly decreases on }] M^{*}, \infty[.\right.
\end{aligned}
$$

Under these assumptions, we have the following result.

Theorem 3.11. Assume that $h_{f}$ is decreasing (2.1), and Assumption I to IV are satisfied then

1- For all $f$, there exists $T_{f}<\infty$ (depending on $\left.\left(\mathcal{M}_{f}(0), \rho_{f}(0)\right)\right)$ s.t. $\left(\mathcal{M}_{f}(t), \rho_{f}(t)\right) \in$ $\Pi_{\text {Differentiated }}$ for all $t>T_{f}$.

2- If $\zeta_{0}^{\infty}>\gamma_{r}$, there exists $\rho^{*}$ s.t., a follicle with a mass $\rho_{f}^{E}>\rho^{*}$ at the entrance in the saved zone is insensitive to the pressure.

3- If $\inf _{\mathcal{M}_{f}, \mathcal{M}} \zeta_{0}\left(\mathcal{M}_{f}, \mathcal{M}\right)<\gamma_{r}$ then there exists $0<\rho_{*}<\infty$ s.t., a follicle with a mass $\rho_{f}^{E}<\rho_{*}$ at the entrance in the saved zone is doomed by the pressure.

4-If $C_{p}=0$ then a follicle is insensitive to the pressure or doomed by the pressure.

3.3. Proof of theorem 3.11. The point 4 - is direct and means that, in the case $C_{p}=0$, the stable set is structurally stable with respect to the exogenous pressure $\mathcal{M}$ or there is a possibility of the follicle to enter again in the sensitive zone (if the pressure is large enough). The dynamic is quite simple. To prove the point $2-$ (resp. $3-$ ), we observe the geometry of the steady states in the paragraph 3.3.1, it suffices then to use the assumption $\zeta_{0}^{\infty}>\gamma_{r}\left(\operatorname{resp} . \inf _{\mathcal{M}_{f}, \mathcal{M}} \zeta_{0}\left(\mathcal{M}_{f}, \mathcal{M}\right)<\gamma_{r}\right)$ and the results of the proposition 3.13 to conclude. Then, by studying the dynamic of the system in paragraph 3.3.2, we prove the point 1 - in the proposition 3.14 and we give bifurcation diagrams to understand the dynamical behavior in the follicle selection process.

Remark. If $\inf _{\mathcal{M}_{f}, \mathcal{M}} \zeta_{0}\left(\mathcal{M}_{f}, \mathcal{M}\right)>\gamma_{r}$, then it is impossible for a follicle in the saved zone to enter again in the sensitive zone. If $\zeta_{0}^{\infty}<\gamma_{r}$, it is impossible for 'large' follicle to enter in the saved zone.

3.3.1. Proof of result 2-, 3-, 4-.. Using assumptions (3.17) and (2.4), the definitions of $\Omega_{\text {lim }}$ (3.9) and $\Pi_{\text {Saved }}(3.5)$, we find that the steady states set belongs to

$$
\text { Steady states set } \subset \Omega_{\text {lim }} \bigcap \Pi_{\text {Saved }} \text {. }
$$

Thus, the geometry of the steady states depends on the maturity speed rate $h_{f}$. Assumptions (3.17), (3.18), (3.19) and technical assumption (3.20) imply that for all $\mathcal{M}$, the graph of $m \mapsto \rho_{0}(m, \mathcal{M})$ looks like figure 3.8. More precisely, we have the following propositions.

Proposition 3.12. Under assumptions (2.1), (3.18) and (3.19), we have the following results

1) Let $\mathcal{D}_{\alpha}=\{(\mathcal{M}, \rho): \rho=\alpha \mathcal{M}\}$ with $\alpha=\rho_{f} / \mathcal{M}_{f}>0$ and $\mathbf{n}_{\alpha}=\left(\begin{array}{c}\alpha \\ -1\end{array}\right) a$ 
normal vector to $\mathcal{D}_{\alpha}$ then we have

$$
\operatorname{sgn}\left(\left.\mathbf{n}_{\alpha} \cdot \frac{\partial}{\partial t}\left(\begin{array}{c}
\mathcal{M}_{f} \\
\rho_{f}
\end{array}\right)\right|_{\rho_{f} / \mathcal{M}_{f}=\alpha}\right)=\operatorname{sgn} h_{f}
$$

2) We have

$$
\left|\rho_{0}(m, 0)-\rho_{0}(m, \infty)\right| \rightarrow_{m \rightarrow \infty} 0 .
$$

3) For all $\mathcal{M}_{f}$, the function $\mathcal{M} \mapsto \rho_{0}\left(\mathcal{M}_{f}, \mathcal{M}\right)$ is strictly increasing (see fig. 3.9).
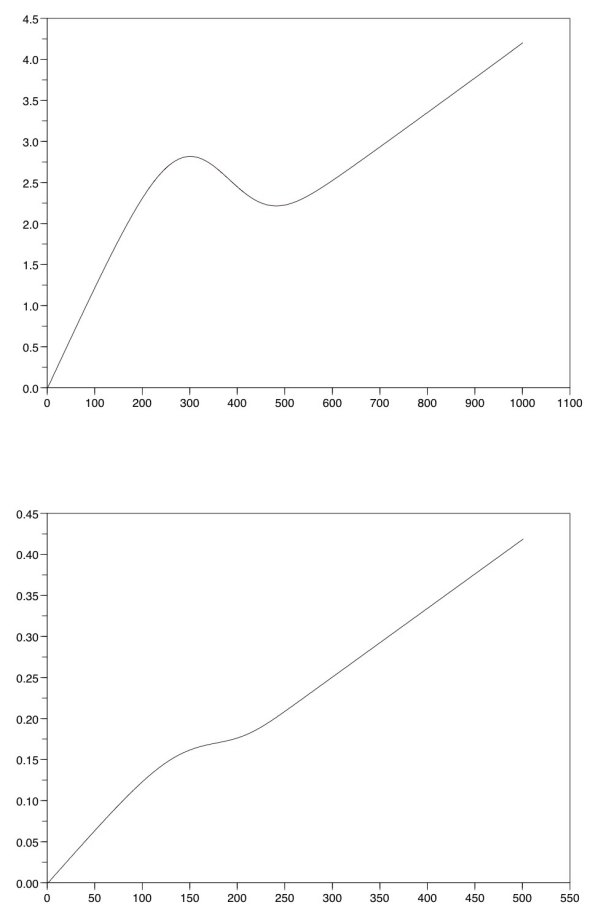

FIG. 3.8. plot of $m \mapsto \rho_{0}(m, \mathcal{M})$ ( $\mathcal{M}$ is fixed) when $C_{p}=1$ and $C_{p}=0$.

Proof. Using (3.19), we have directly (3.24). In the same way, 3) comes directly from assumption (3.18). Now, using (3.1), we find that

$$
\left(\begin{array}{c}
\alpha \\
-1
\end{array}\right) \cdot \frac{\partial}{\partial t}\left(\begin{array}{c}
\mathcal{M}_{f} \\
\rho_{f}
\end{array}\right)=\alpha\left(h_{f} \rho_{f}+\Delta \mathcal{M}_{f}\right)-\Delta \rho_{f}=\alpha h_{f} \rho_{f}
$$

since $\alpha \geq 0$, we finally obtain that (3.23) is satisfied.

口

Proposition 3.13. Under assumptions (2.1), (3.17)-(3.19) and technical assumptions (3.20), we have the number of connex component of the intersection of $\Gamma_{\rho_{0}}(\mathcal{M})$ and the horizontal line

$$
\mathcal{D}\left(\rho_{d}\right)=\left[0, \infty\left[\times\left\{\rho_{d}\right\},\right.\right.
$$




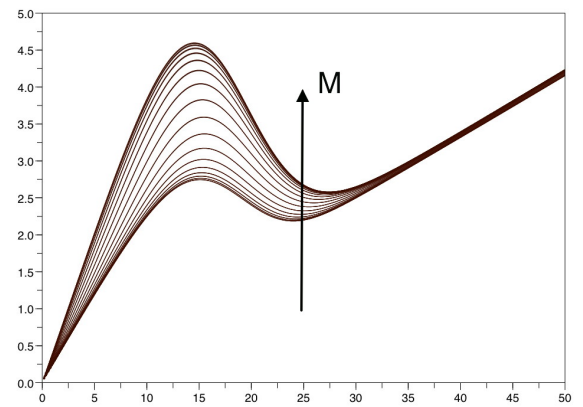

FIG. 3.9. plot of $m \mapsto \rho_{0}(m, \mathcal{M})$ (for several $\left.\mathcal{M}\right)$.

which statisfies,

1) The horizontal line $\left[0, \infty\left[\times\left\{\rho_{d}\right\}\right.\right.$ intersects the curve given by the function $\rho_{0}$ in less than 3 connex components

$$
\forall \mathcal{M}, \rho_{d} \in\left[0, \infty\left[, \quad N\left(\rho_{d} \mid \mathcal{M}\right) \leq 3 .\right.\right.
$$

2) There exists $\rho_{d}^{*}>0$ s.t.

$$
\forall \mathcal{M} \geq 0, \rho_{d}>\rho_{d}^{*}, \quad N\left(\rho_{d} \mid \mathcal{M}\right)=1 .
$$

3) There exists $\rho_{d *}>0$ s.t.

$$
\forall \mathcal{M} \geq 0, \rho_{d}<\rho_{d *}, \quad N\left(\rho_{d} \mid \mathcal{M}\right)=1 .
$$

4) Moreover, if we have

$$
\max _{\mathcal{M}_{f}, \mathcal{M}} \mathcal{M}_{f} \frac{\partial}{\partial \mathcal{M}_{f}} \log \left(\zeta_{0}\right)\left(\mathcal{M}_{f}, \mathcal{M}\right) \leq 1 \text {, i.e. } C_{p}=0
$$

then $\rho_{d *}=\infty$.

Proof. To prove this proposition, we fix $\mathcal{M}$ and we first compute $\frac{\partial}{\partial \mathcal{M}_{f}} \rho_{0}$,

$$
\frac{\partial}{\partial \mathcal{M}_{f}} \rho_{0}\left(\mathcal{M}_{f}, \mathcal{M}\right)=\frac{\partial}{\partial \mathcal{M}_{f}} \mathcal{M}_{f} / \zeta_{0}\left(\mathcal{M}_{f}, \mathcal{M}\right)=\frac{1}{\zeta_{0}}\left[1-\mathcal{M}_{f} \frac{\partial}{\partial \mathcal{M}_{f}} \log \left(\zeta_{0}\right)\right] .
$$

The sign of $\frac{\partial}{\partial \mathcal{M}_{f}} \rho_{0}$ only depends on the sign of $1-\mathcal{M}_{f} \frac{\partial}{\partial \mathcal{M}_{f}} \log \left(\zeta_{0}\right)$. Under the assumptions (3.20), we have two cases : we fix the pressure $\mathcal{M} \geq 0$.

Case 1: Increasing.. If we have

$$
\mathcal{M}_{f} \frac{\partial}{\partial \mathcal{M}_{f}} \log \left(\zeta_{0}\right) \leq 1, \quad \forall \mathcal{M}_{f},
$$

then $\rho_{0}$ is continuous and increasing (not necessarily strictly) and we have that $\rho_{0}(0,)=0,. \rho_{0}(\infty,)=.\infty$ (using (3.17)). Therefore, for all $\rho_{d} \geq 0, \mathcal{M} \geq 0$, we find that the number of connex comp. of $\mathcal{D}\left(\rho_{d}\right) \cap \Gamma_{\rho_{0}}(\mathcal{M})$ is equal to 1 , which proves point 4). 

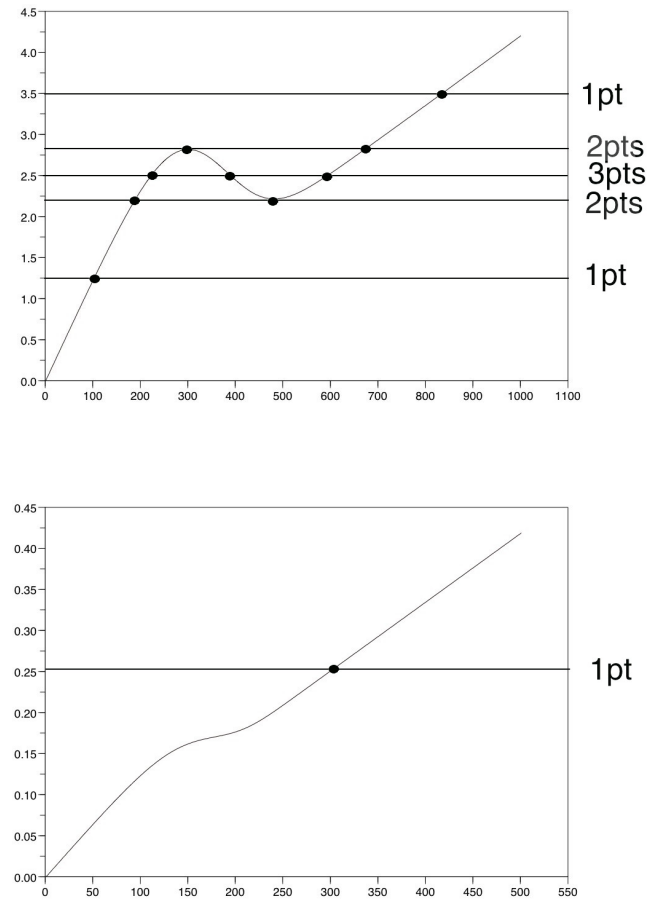

FIG. 3.10. plot of $m \mapsto \rho_{0}(m, \mathcal{M})\left(\mathcal{M}\right.$ is fixed) and the line $\mathcal{D}\left(\rho_{d}\right)$ for several $\rho_{d}$.

Case 2 : Increasing-Decreasing-Increasing.. If there exists $\mathcal{M}_{f}$ so that

$$
\mathcal{M}_{f} \frac{\partial}{\partial \mathcal{M}_{f}} \log \left(\zeta_{0}\right)>1
$$

then $\left.\left\{\mathcal{M}_{f}: \mathcal{M}_{f} \frac{\partial}{\partial \mathcal{M}_{f}} \log \left(\zeta_{0}\right) \geq 1\right\}=\left[\underline{M}_{f}(\mathcal{M}), \bar{M}_{f}(\mathcal{M})\right] \subset\right] 0, \infty\left[\right.$, and $\rho_{0}$ is strictly increasing on $\left[0, \underline{M}_{f}(\mathcal{M})\right]$, decreasing (not necessarily strictly) on $\left[\underline{M}_{f}(\mathcal{M}), \bar{M}_{f}(\mathcal{M})\right]$ and strictly increasing on $\left[\bar{M}_{f}(\mathcal{M}), \infty\left[\right.\right.$ with $\rho_{0}(0,)=0,. \rho_{0}(\infty,)=.\infty$ (using $\left.(3.17)\right)$. Therefore, using the strict growth of $\rho_{0}$, we find that

$$
\begin{gathered}
{\left[0, \underline{M}_{f}\left[\times\left\{\rho_{d}\right\} \bigcap \Gamma_{\rho_{0}}(\mathcal{M})\right. \text { is a single point (or empty), }\right.} \\
] \bar{M}_{f}, \infty\left[\times\left\{\rho_{d}\right\} \bigcap \Gamma_{\rho_{0}}(\mathcal{M})\right. \text { is a single point (or empty), }
\end{gathered}
$$

and using that $\rho_{0}$ is decreasing, we have

$$
\left[\underline{M}_{f}, \bar{M}_{f}\right] \times\left\{\rho_{d}\right\} \bigcap \Gamma_{\rho_{0}}(\mathcal{M}) \text { is an interval (or empty). }
$$

Therefore $N\left(\rho_{d} \mid \mathcal{M}\right) \leq 3$ and we have, more precisely,

$$
N\left(\rho_{d} \mid \mathcal{M}\right)=1, \quad \forall \rho_{d}<\rho_{0}\left(\bar{M}_{f}, \mathcal{M}\right),
$$




$$
N\left(\rho_{d} \mid \mathcal{M}\right)=1, \quad \forall \rho_{d}>\rho_{0}\left(\underline{M}_{f}, \mathcal{M}\right),
$$

and

$$
\begin{aligned}
& \left.N\left(\rho_{d} \mid \mathcal{M}\right)=3, \quad \forall \rho_{d} \in\right] \rho_{0}\left(\bar{M}_{f}, \mathcal{M}\right), \rho_{0}\left(\underline{M}_{f}, \mathcal{M}\right)[, \\
& N\left(\rho_{d} \mid \mathcal{M}\right)=2, \text { as } \rho_{d}=\rho_{0}\left(\bar{M}_{f}, \mathcal{M}\right), \rho_{0}\left(\underline{M}_{f}, \mathcal{M}\right) .
\end{aligned}
$$

We then have proved (3.26), (3.27) and (3.28). It suffices to let

$$
\rho_{d}^{*}=\min _{\mathcal{M}} \rho_{0}\left(\bar{M}_{f}(\mathcal{M}), \mathcal{M}\right), \quad \rho_{d *}=\max _{\mathcal{M}} \rho_{0}\left(\underline{M}_{f}(\mathcal{M}), \mathcal{M}\right) .
$$

Consequently, we have directly the point 4- (resp. 2- and 3-) of theorem 3.11 given by the point 4) (resp. 2) and 3)) of proposition 3.13.

3.3.2. Proof of 1-. and bifurcation diagram. We study the dynamic in both, proliferation (3.2) and differentiated zone (3.3). We first notice that the proliferation zone is a transient set, which means that a follicle must escape this zone in a finite time. At the end of this process, there is two possibilities, the follicle may stay in the sensitive zone and die (atresia [5]) or escape the sensitive zone. As long as the follicle stay in the saved zone (3.5), the mass of the follicle is constant, equal to the mass at the entrance in the saved zone : $\rho_{f}^{E}$. There are several possibilities, the mass $\rho_{f}^{E}$ is large enough and the follicle is insensitive to the pressure exerted by the others follicles (3.14), this is the best case, its steady states set is structurally stable with respect to the exogenous pressure. If the mass $\rho_{f}^{E}$ is too small, then the follicle is doomed by the pressure (3.15). This is the worst case, under an exogenous pressure large enough (if a follicle, or several follicles are mature enough), there is no steady state in the saved zone and the follicle must enter again in the sensitive zone and loose mass. If the mass $\rho_{f}^{E}$, is large but not too large, the dynamic is much more complex : it can reach a structurally stable state, enter again in the sensitive zone or oscillate (hard case to obtain).

The Proliferation Zone : a transient set. There exists a maximal time $T^{*}>0$, depending on the initial data $\zeta_{0}\left(\mathcal{M}_{f}(t=0), \mathcal{M}(t=0)\right)$,

$$
T^{*}=\frac{\gamma_{s}-\zeta_{0}\left(\mathcal{M}_{f}(t=0), \mathcal{M}(t=0)\right)}{\inf _{m, M, x \leq \gamma_{s}} h_{f}(m, M, x)}<\infty,
$$

so that

$$
\forall t \geq T^{*}\left(\mathcal{M}_{f}, \rho_{f}\right)(t) \in \Pi_{\text {Differentiated }}
$$

Proposition 3.14. Under assumption (3.17), we find that (3.31) is satisfied with $T^{*}$ defined in (3.30).

Proof. It suffices to notice that $q(t)=\left(\gamma_{s}-\zeta_{f}(t)\right)$ satisfies $q^{\prime}(t)=-h_{f}\left(\mathcal{M}_{f}, \mathcal{M}, \zeta_{f}(t)\right)$. Indeed assuming that $\zeta_{f}(t)<\gamma_{s}$ for all $t>0$ and using (3.17) we have that $q^{\prime}(t) \leq$ $-\inf _{m, M, x \leq \gamma_{s}} h_{f}(m, M, x)<0$. Thus, there exists $t_{s}<T^{*}$ so that $q\left(t_{s}\right)=0$ and for all $t \geq t_{s}$, we have $\zeta_{f}(t)>\gamma_{s}$ and (3.31) is verified. $\square$

We notice that assumption (3.18) implies that $M \mapsto \inf _{m, x \leq \gamma_{s}} h_{f}(m, M, x)$ decreases. Therefore, the time that a follicle stay in the proliferation zone may depend on the maturity of all the others follicles. 
Differentiated and Saved Zone : a crucial zone. We remark that, in the differentiated zone, the mass of each follicles is decreasing and so convergent, thus the dynamic of the system is only driven by the maturity speed rate $h_{f}$. Moreover, we notice that the dynamic of a follicle depends on the pressure of the others follicles. So it is interesting to study the bifurcation as follows, given a mass $\rho_{f}^{E}$ at the entrance in the saved zone, the steady states of the maturity $\mathcal{M}_{f}$ depend on the maturity pressure $\mathcal{M}$. Under assumption (3.20), we let, when $C_{p} \neq 0$, (see fig. 3.11 and 3.12)

$$
\left.\left\{\mathcal{M}_{f}: \mathcal{M}_{f} \frac{\partial}{\partial \mathcal{M}_{f}} \log \left(\zeta_{0}\right) \geq 1\right\}=\left[\underline{M}_{f}(\mathcal{M}), \bar{M}_{f}(\mathcal{M})\right] \subset\right] 0, \infty[
$$

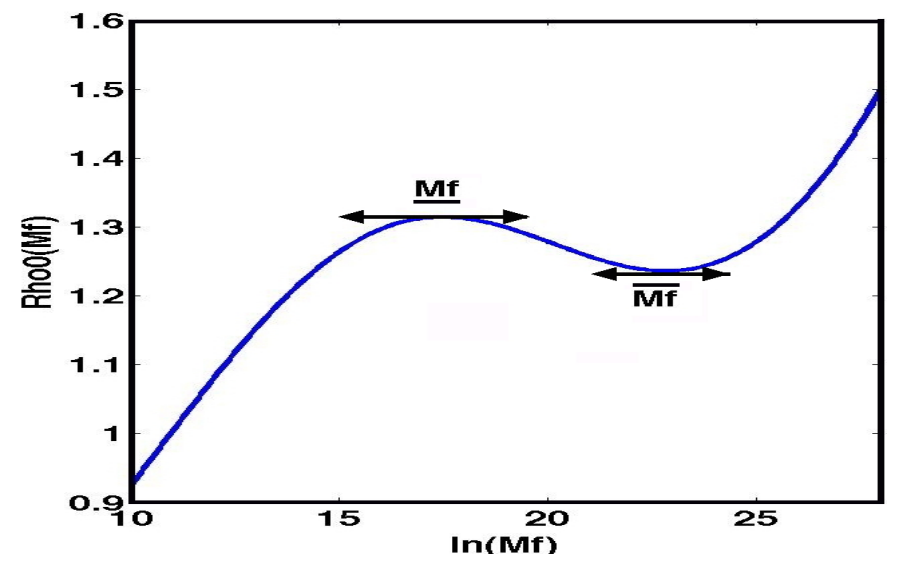

FIG. 3.11. $\mathcal{M}$ is fixed, then $\underline{M}_{f}(\mathcal{M}), \bar{M}_{f}(\mathcal{M})$ are the critical points of $m \rightarrow \rho_{0}(m, \mathcal{M})$.

$$
\begin{aligned}
& \bar{\rho}_{\text {max }}=\max _{\mathcal{M}} \rho_{0}\left(\underline{M}_{f}(\mathcal{M}), \mathcal{M}\right), \quad \underline{\rho}_{\text {max }}=\min _{\mathcal{M}} \rho_{0}\left(\underline{M}_{f}(\mathcal{M}), \mathcal{M}\right), \\
& \bar{\rho}_{\text {min }}=\max _{\mathcal{M}} \rho_{0}\left(\bar{M}_{f}(\mathcal{M}), \mathcal{M}\right), \quad \underline{\rho}_{\text {max }}=\min _{\mathcal{M}} \rho_{0}\left(\bar{M}_{f}(\mathcal{M}), \mathcal{M}\right) .
\end{aligned}
$$

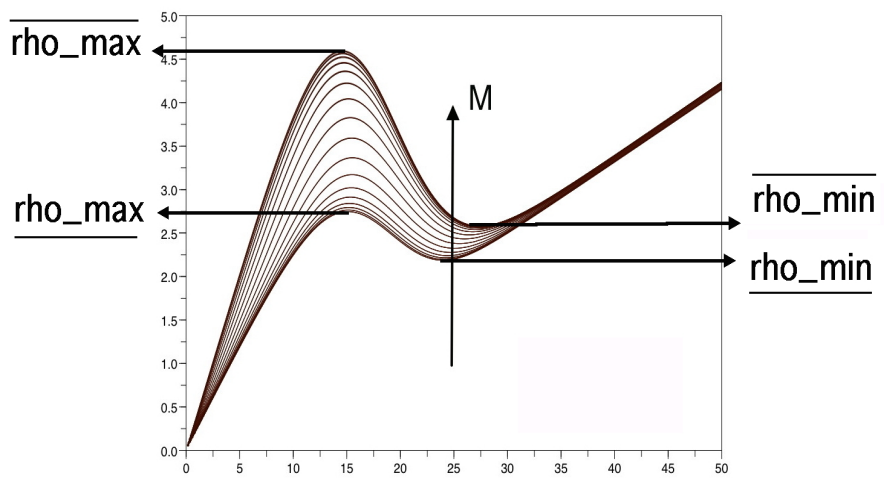

FIG. 3.12. $\bar{\rho}_{\max }, \underline{\rho}_{\max }, \quad \bar{\rho}_{\min }$ and $\underline{\rho}_{\max }$. 
Firstly, we plot the bifurcation diagram, $\mathcal{M} \mapsto \rho_{0}(., \mathcal{M})^{-1}\left(\rho_{f}^{E}\right)$, i.e., $\mathcal{M} \mapsto\left\{\mathcal{M}_{f}\right.$ : $\left.\rho_{0}\left(\mathcal{M}_{f}, \mathcal{M}\right)=\rho_{f}^{E}\right\}$. To avoid the multiplication of cases, we do not take in account the sensitive zone, i.e. the possibility of a follicle to enter again in the sensitive zone. To take in account the sensitive zone, it suffices to draw a straight horizontal line $\mathcal{M}_{f}=\gamma_{r} \rho_{f}^{E}$ and say that there is no steady states under this line. We study the bifurcations in different cases:

- when $\rho_{f}^{E}$ is large enough $\rho_{f}^{E}>\bar{\rho}_{\text {max }}$, the steady state set is structurally stable with respect to the pressure $\mathcal{M}$ (see figure 3.13),

- when $\rho_{f}^{E}$ is too small, $\rho_{f}^{E}<\underline{\rho}_{\min }$, the steady state set is structurally stable with respect to the pressure $\mathcal{M}$ (see figure 3.13 ),

- in other cases appears bifurcations and catastrophes.
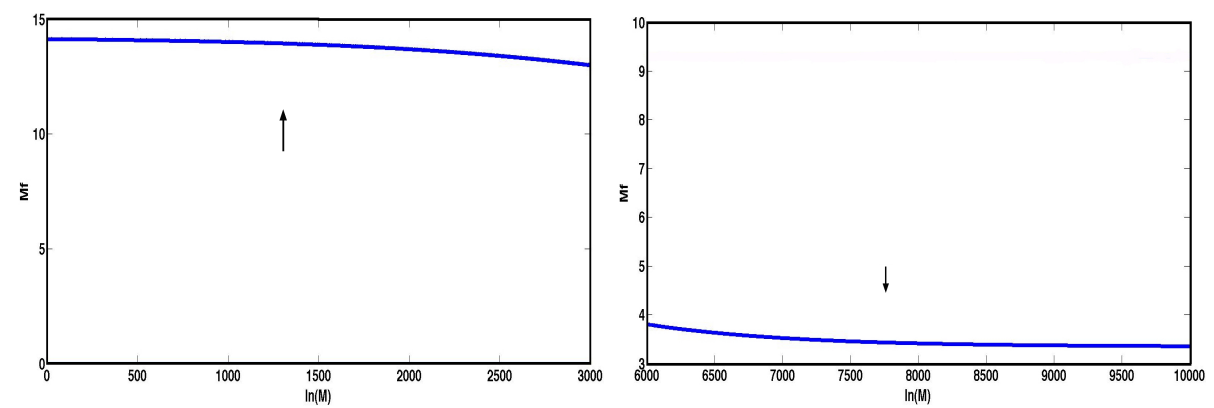

FIG. 3.13. $\rho_{f}^{E}>\bar{\rho}_{\max }$ (left), $\quad \rho_{f}^{E}<\underline{\rho}_{\min }($ right $)$, we plot $\left(\mathcal{M},\left\{\mathcal{M}_{f}: \rho_{0}\left(\mathcal{M}_{f}, \mathcal{M}\right)=\rho_{f}^{E}\right\}\right)$.

We explain here, how to read the figure 3.13, imagine that a follicle $f$ enter in the saved zone with a mass $\rho_{f}^{E}>\bar{\rho}_{\text {max }}$, then whatever the maturity of all the other follicles is, the follicle $f$ will reach a maturity $\left\{\mathcal{M}_{f}: \rho_{0}\left(\mathcal{M}_{f}, \mathcal{M}\right)=\rho_{f}^{E}\right\}$ which softly depends on the maturity $\mathcal{M}$. In figure 3.14 , we imagine that the maturity $\mathcal{M}$ evolves (increases, decreases and increases), the follicle $f$ enters in the saved zone with $\rho_{f}^{E}>\bar{\rho}_{\text {max }}$ and a maturity $\rho_{f}^{E} \gamma_{s}$, then we see that the maturity $\mathcal{M}_{f}$ is "attracted" by the curve following the arrow.

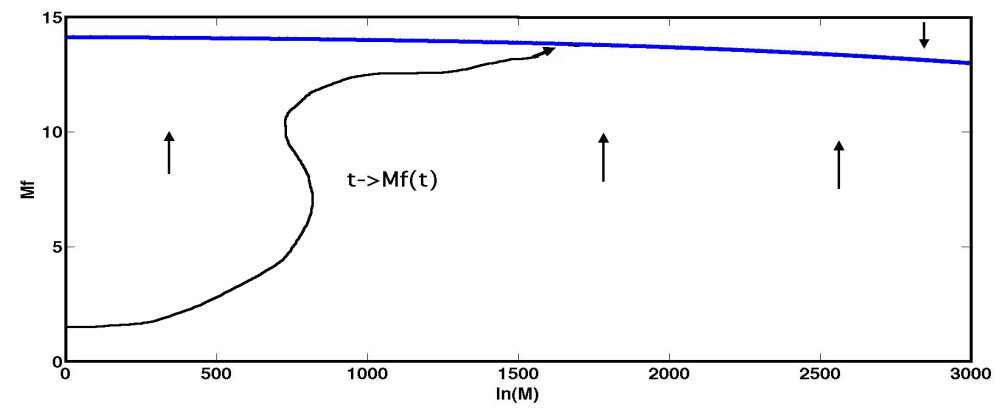

FIG. 3.14. $\rho_{f}^{E}>\bar{\rho}_{\text {max }}$ we see the evolution of $t \rightarrow \mathcal{M}_{f}(t)$ when $\mathcal{M}$ evolves (increases, decreases and increases). 
We plot the bifurcation diagram, $\mathcal{M} \mapsto \rho_{0}(., \mathcal{M})^{-1}\left(\rho_{f}^{E}\right)$, i.e., $\mathcal{M} \mapsto\left\{\mathcal{M}_{f} \quad:\right.$ $\left.\rho_{0}\left(\mathcal{M}_{f}, \mathcal{M}\right)=\rho_{f}^{E}\right\}$ when we have $\underline{\rho}_{\text {max }}<\bar{\rho}_{\text {min }}$.
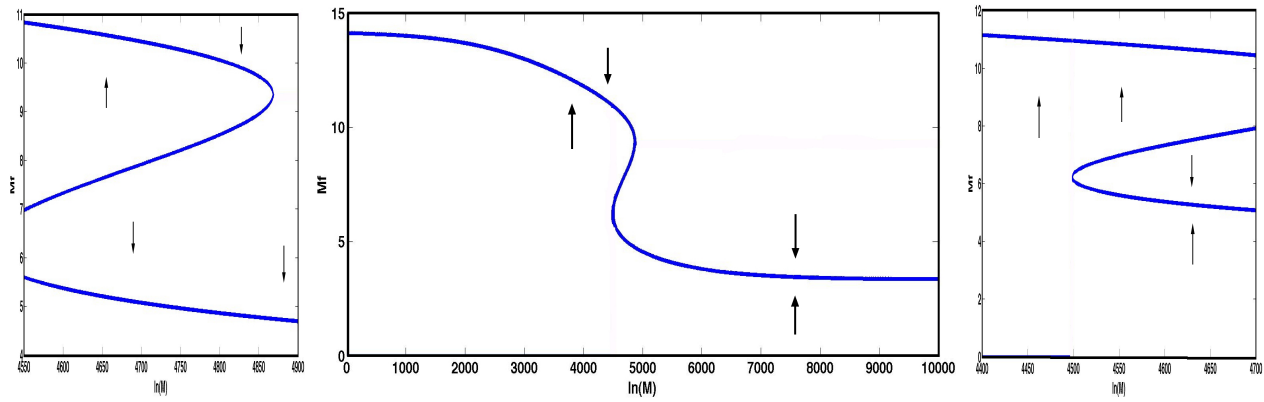

FIG. 3.15. $\underline{\rho}_{\text {min }}<\rho_{f}^{E}<\underline{\rho}_{\max }, \quad \underline{\rho}_{\text {max }}<\rho_{f}^{E}<\bar{\rho}_{\text {min }}, \quad \bar{\rho}_{\text {min }}<\rho_{f}^{E}<\bar{\rho}_{\text {max }}$

We explain here, how to read the figure 3.15 left bifurcation diagram, imagine that a follicle $f$ enter in the saved zone with a mass $\underline{\rho}_{\min }<\rho_{f}^{E}<\underline{\rho}_{\max }$ with $\underline{\rho}_{\max }<\bar{\rho}_{\min }$, then :

- if the maturity of all the other follicles $\mathcal{M}$ is small enough then the follicle $f$ will reach a large maturity (upper part of the curve),

- if the maturity of all the other follicles $\mathcal{M}$ is large enough then the follicle $f$ will reach a small maturity (lower part of the curve).

In figure 3.16, we imagine that the maturity $\mathcal{M}$ slowly increases, the follicle $f$ enters in the saved zone with $\underline{\rho}_{\min }<\rho_{f}^{E}<\underline{\rho}_{\max }$ and a maturity $\rho_{f}^{E} \gamma_{s}$, then we see that the maturity $\mathcal{M}_{f}$ is "attracted" by the upper part of the curve (following the arrow) nevertheless if the maturity $\mathcal{M}$ increases too much then $\mathcal{M}_{f}$ falls from the upper part to the lower part of the curve (there is a catastrophe) and then whatever $\mathcal{M}$ becomes, the follicle $f$ will stay with a low maturity. Here we understand, why we say that in this case the follicle $f$ is "sensitive" to the pressure of the other follicles.

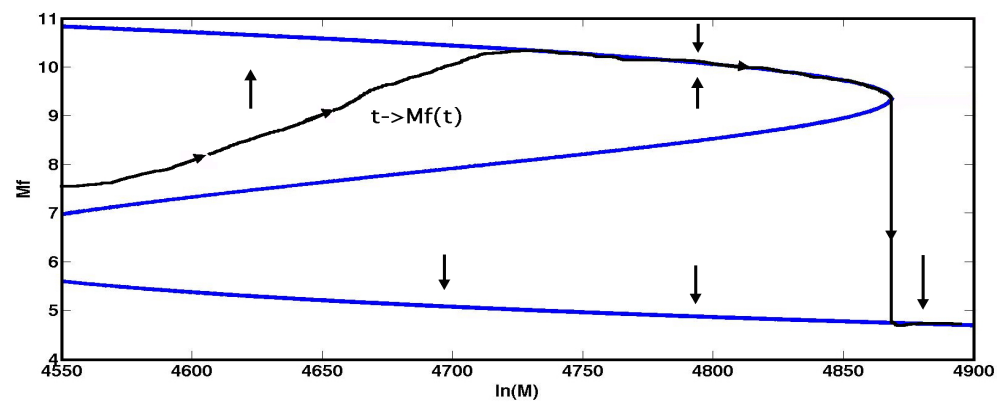

FIG. 3.16. $\underline{\rho}_{\min }<\rho_{f}^{E}<\underline{\rho}_{\max }$ when $\underline{\rho}_{\max }<\bar{\rho}_{\text {min }}$ and we see the evolution of $t \rightarrow \mathcal{M}_{f}(t)$ when $\mathcal{M}$ increases. 
We plot the bifurcation diagram, $\mathcal{M} \mapsto \rho_{0}(., \mathcal{M})^{-1}\left(\rho_{f}^{E}\right)$, i.e., $\mathcal{M} \mapsto\left\{\mathcal{M}_{f} \quad:\right.$ $\left.\rho_{0}\left(\mathcal{M}_{f}, \mathcal{M}\right)=\rho_{f}^{E}\right\}$ when we have $\bar{\rho}_{\text {min }}<\underline{\rho}_{\text {max }}$.

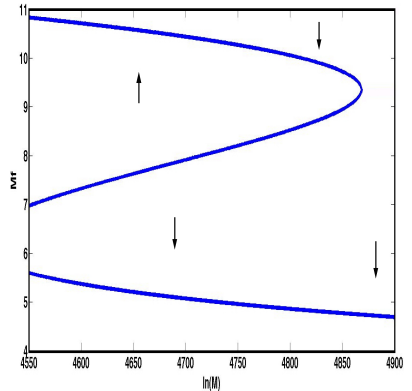

FIG. 3.17. $\underline{\rho}_{\min }<\rho_{f}^{E}<\bar{\rho}_{\min }$,

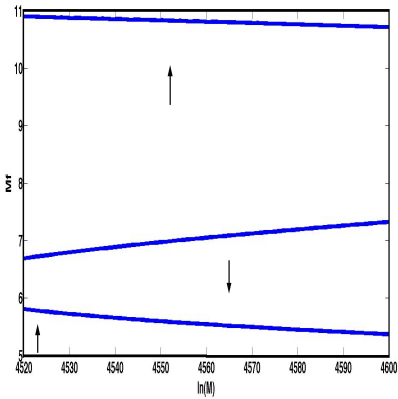

$\bar{\rho}_{\min }<\rho_{f}^{E}<\underline{\rho}_{\max }$,

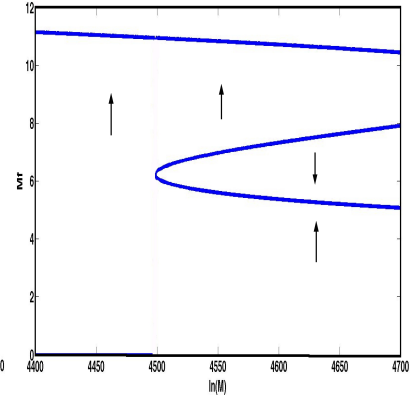

$\underline{\rho}_{\max }<\rho_{f}^{E}<\bar{\rho}_{\max }$

We explain here, how to read the figure 3.17 right bifurcation diagram, imagine that a follicle $f$ enter in the saved zone with a mass $\underline{\rho}_{\max }<\rho_{f}^{E}<\bar{\rho}_{\max }$ with $\bar{\rho}_{\text {min }}<\underline{\rho}_{\max }$, then :

- if the maturity of all the other follicles $\mathcal{M}$ is small enough then the follicle $f$ will reach a large maturity (upper part of the curve),

- if the maturity of all the other follicles $\mathcal{M}$ is large enough then the follicle $f$ will reach a small maturity (lower part of the curve).

In figure 3.18 , we imagine that the maturity $\mathcal{M}$ slowly increases, the follicle $f$ enters in the saved zone with $\underline{\rho}_{\max }<\rho_{f}^{E}<\bar{\rho}_{\max }$ and a maturity $\rho_{f}^{E} \gamma_{s}$, then we see that two cases can appear :

- the follicle $f$ reach large maturity before that the maturity of all the other follicles $\mathcal{M}$ becomes too large and then the follicle $f$ has its maturity which stays in the upper part of the curve, i.e., is not sensitive to the variation of $\mathcal{M}$,

- the follicle $f$ does not reach large maturity before that the maturity of all the other follicles $\mathcal{M}$ becomes too large and then the follicle $f$ has its maturity which stays in the lower part of the curve (low maturity follicle, maybe doomed follicle).

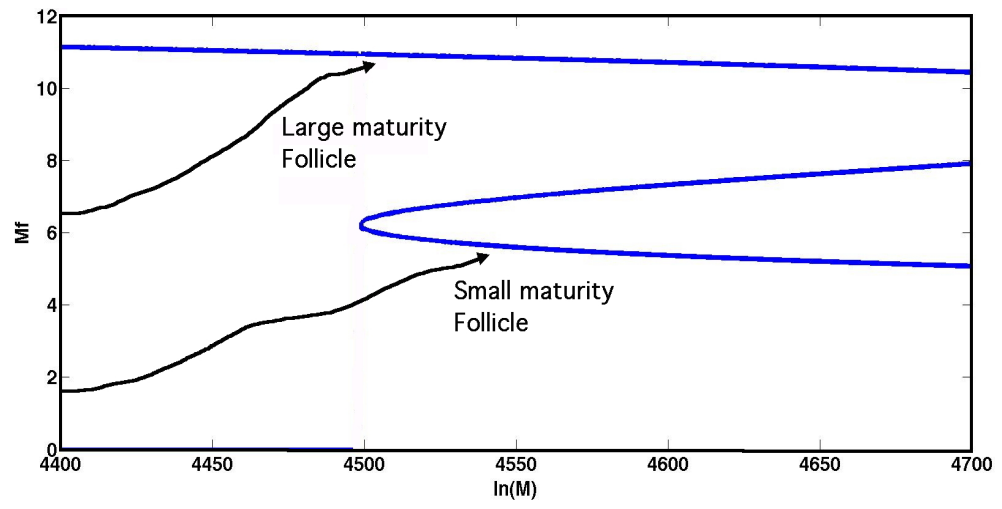

FIG. 3.18. $\underline{\rho}_{\text {min }}<\rho_{f}^{E}<\underline{\rho}_{\max }$ when $\bar{\rho}_{\text {min }}<\underline{\rho}_{\max }$ and we see the evolution of $t \rightarrow \mathcal{M}_{f}(t)$ when $\mathcal{M}$ increases. 
Proposition 3.15. Under assumption (2.1), (3.17)-(3.20), and $\underline{\rho}_{\min }<\bar{\rho}_{\min }<$ $\underline{\rho}_{\max }<\bar{\rho}_{\max }$ (resp. $\underline{\rho}_{\min }<\underline{\rho}_{\max }<\bar{\rho}_{\min }<\bar{\rho}_{\max }$ ) bifurcation diagram are given by figure 3.17 and figure 3.13 (resp. figure 3.15, figure 3.13 and, when there exists $\mathcal{M}$ s.t. $\rho_{0}\left(\underline{M}_{f}(\mathcal{M}), \mathcal{M}\right)=\rho_{0}\left(\bar{M}_{f}(\mathcal{M}), \mathcal{M}\right)$, figure 3.20 and 3.19).

Remark. In the case when there exists $\mathcal{M}$ s.t. $\rho_{0}\left(\underline{M}_{f}(\mathcal{M}), \mathcal{M}\right)=\rho_{0}\left(\bar{M}_{f}(\mathcal{M}), \mathcal{M}\right)$, i.e., $\rho_{0}(., \mathcal{M})$ is increasing, then can appear jumps as in figure 3.20 and 3.19 .
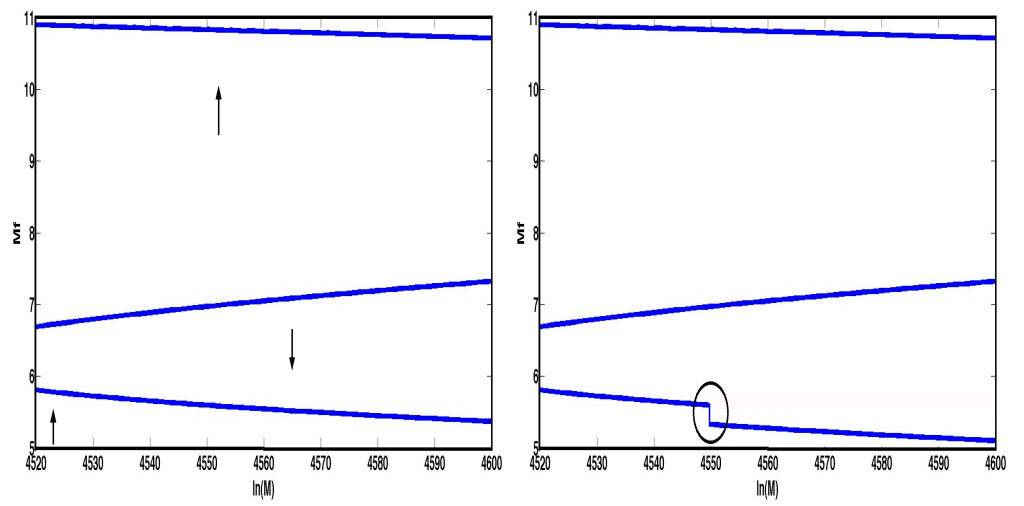

FIG. 3.19. $\bar{\rho}_{\min }<\rho_{f}^{E}<\underline{\rho}_{\max }$

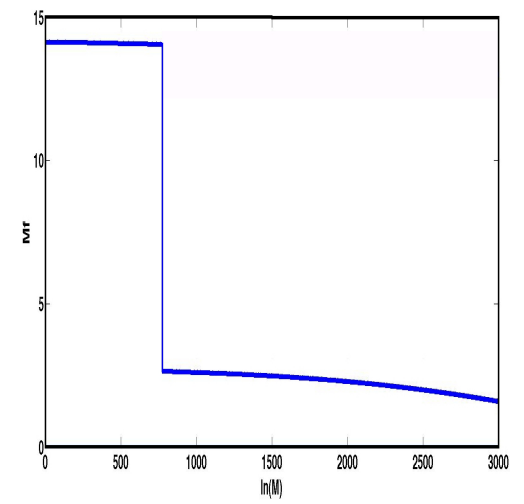

FIG. 3.20. $\rho_{f}^{E}=\rho_{0}\left(\underline{M}_{f}(\mathcal{M}), \mathcal{M}\right)=\rho_{0}\left(\bar{M}_{f}(\mathcal{M}), \mathcal{M}\right)$

Proposition 3.16. Under assumption (2.1), (3.17)-(3.20) and $C_{p}=0$, i.e., $\rho_{0}(., \mathcal{M})$ is increasing for all $\mathcal{M}$, then the bifurcation diagram is given by figure 3.20 or figure 3.13.

4. Numerical simulation and comparison to the Lacker Model. To simulate the simplified system of ODE (3.1) giving the evolution of the mass and maturity of each follicle in a pool of $N$ follicles, we have developed a Mac OS software (ProjetFolliV2, see : http://perso.ec-lyon.fr/michel.philippe/ProjetFolliV2.zip) using an Euler scheme (sufficient, here, to observe the dynamic of this system of ODE). This software shows the evolution of a pool (up to 60) follicles in the proliferation-sensitive and differentiated zone. The death rate, birth rate, global and local control are given 
by the F. Clement paper [14] : the authors define the maturity speed rate

$$
h_{f}\left(u_{f}, \zeta(t)\right)=0.07\left(-\zeta^{2}+(11.892 \zeta+2.888)\left(1-e^{-u_{f} / 0.133}\right)\right),
$$

with the local feedback control

$$
u_{f}\left(\mathcal{M}_{f}, \mathcal{M}\right)=\left(0.054+\frac{1-0.054}{1+e^{0.3\left(27-\mathcal{M}_{f}\right)}}\right) U\left(t, \mathcal{M}_{T}\right),
$$

and the global feedback control

$$
U(t, \mathcal{M})=\left(0.5+\frac{0.5}{1+e^{0.1\left(\mathcal{M}_{T}-50\right)}}\right)+U_{0}(t) .
$$

Here $U_{0}$ which corresponds to a potential exogenous entry of FSH. The birth rate is defined as follows

$$
B\left(\mathcal{M}_{f}, \mathcal{M}_{T}, \mathcal{M}_{f} / \rho_{f}\right)=(\ln (2) / 2)\left(1-0.5\left(1-u_{f}\left(\mathcal{M}_{f}, \mathcal{M}_{T}\right)\right) 1_{\mathcal{M}_{f} / \rho_{f}<\gamma_{s}}\right),
$$

and the death rate is equal to

$$
(4.5) \Lambda\left(\mathcal{M}_{f}, \mathcal{M}_{T}, \mathcal{M}_{f} / \rho_{f}\right)=3 e^{-\frac{\left(\mathcal{M}_{f} / \rho_{f}-\gamma_{s}\right)^{2}}{0.2^{2}}}\left(1-U\left(\mathcal{M}_{T}\right)\right) 1_{\mathcal{M}_{f} / \rho_{f} \in\left[\gamma_{s}-0.5, \gamma_{s}+0.5\right]} .
$$

Therefore we have a global growth rate $\Delta\left(\mathcal{M}_{f}, \mathcal{M}_{T}, \mathcal{M}_{f} / \rho_{f}\right)$ as

$$
\Delta\left(\mathcal{M}_{f}, \mathcal{M}_{T}, \mathcal{M}_{f} / \rho_{f}\right)=B\left(\mathcal{M}_{f}, \mathcal{M}_{T}, \mathcal{M}_{f} / \rho_{f}\right)-\Lambda\left(\mathcal{M}_{f}, \mathcal{M}_{T}, \mathcal{M}_{f} / \rho_{f}\right) .
$$

The software allows us to change parameters as the age speed rate parameter $\left(\tau_{g}=1\right.$ in [14] $)$, the maturity speed rate parameter $\left(\tau_{h}=0.07\right.$ in [14] $) \ldots$ to see which parameters are important in the dynamic of (3.1). We have directly, through a computation of the zero of the maturity speed rate $h_{f}$ that

Lemma 4.1. The function $h_{f}$ satisfies Assumption I-IV and so the dynamic of the evolution of the pool of follicles is given by bifurcation diagrams (see section 3.3.2) and the results of the theorem 3.11 hold.

Lemma 4.2. There exists $\bar{U}$ s.t. $C_{p}=0$ for an exogenous $F S H U_{0}(t) \geq \bar{U}$.

Proof. We have that

$$
\zeta_{0}\left(\mathcal{M}_{f}, \mathcal{M}\right)=\frac{11.892 r_{f}+\sqrt{\left(11.892 r_{f}^{2}+4 * 2.888 r_{f}\right.}}{2},
$$

with $\left.r_{f}=\left(1-e^{-u_{f} / 0.133}\right)\right)$ and

$$
\left(1-e^{-u_{f} / 0.133}\right)=\left(1-e^{-\left(0.054+\frac{1-0.054}{1+e^{0.3\left(27-\mathcal{M}_{f}\right)}}\right)\left[\left(0.5+\frac{0.5}{1+e^{0.1\left(\mathcal{M}+\mathcal{M}_{f}-50\right)}}\right)+U_{0}(t)\right] / 0.133}\right) .
$$

Thus for $U_{0}$ large enough, we find that

$$
\begin{aligned}
\frac{\partial}{\partial \mathcal{M}_{f}}\left(1-e^{-u_{f} / 0.133}\right) & \sim U_{0} e^{-u_{f} / 0.133} \frac{\partial}{\partial \mathcal{M}_{f}}\left(0.054+\frac{1-0.054}{1+e^{0.3\left(27-\mathcal{M}_{f}\right)}}\right), \\
\zeta_{0}\left(\mathcal{M}_{f}, \mathcal{M}\right) & \sim \frac{11.892+\sqrt{(11.892)^{2}+4 * 2.888}}{2},
\end{aligned}
$$

and so we have $\frac{\partial}{\partial \mathcal{M}_{f}} \zeta_{0}\left(\mathcal{M}_{f}, \mathcal{M}\right)<\zeta_{0}\left(\mathcal{M}_{f}, \mathcal{M}\right) / \mathcal{M}_{f}$ and $C_{p}=0$.

Remark.This means that an exogenous injection of FSH makes the system more stable (structurally stable) and follicles can easily reach a follicular maturity threshold (ovulation with a lots of follicles). 
4.1. A Numerical simulation explained. Let describe the following simulation (see figure 4.1), where we show the maturity $M_{i}$ with respect to the time of four follicles in competition (using an Euler scheme).

- At the beginning, all the follicles are in the proliferation zone and they grow up and became their mean maturity $M_{f}$ increase (due to the growth of the mass and maturity).

- Then, around time $=500$, follicles enter in the sensitive zone and get out of the proliferation zone : the mass decreases and so the mean maturity decreases (even if the local maturity go on increasing).

- Around time $=1000$, follicles get out of the sensitive zone and their mass $\rho$ become constant (unless to enter again in the sensitive zone). Here, the dynamic is given by the equation (2.30) and bifurcation diagrams (see section 3.3.2).

For instance, the more mature follicle follow the second diagram of the figure 3.15, i.e. the follicle enters in the differentiated zone with enough mass, and the less mature follow the second diagram of the figure 3.13, i.e. the follicle enters in the differentiated zone with a too small mass.

- Around time $=3500$ (boxed), we have a bifurcation which appears (see the second diagram of the figure 3.15 when $M$ is smaller and smaller ) : the mean maturity of the more mature follicle is large enough and the mean maturity of all the others follicles is small enough then the more mature follicle reach the first branch (the higher one) and its maturity increases quickly. In the same time, for all other follicle $i \neq 1$ the mean maturity of the others follicles (which include the follicle $j=1$ ) increases and the mean maturity of the follicle $i$ decreases.

- Finally, around time $=4500$, we see that the two less mature follicles which enter again in the sensitive zone and disappear (death by atresia). 


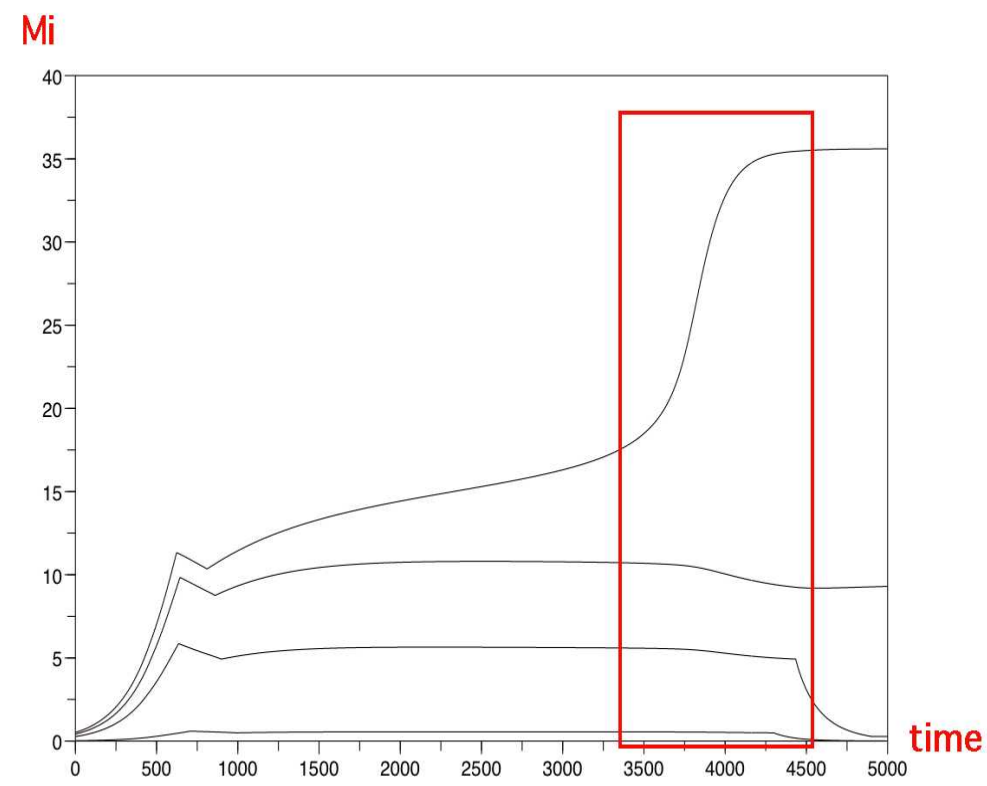

FIG. 4.1. Simulation: Mean Maturity $M_{i}$ with respect to time.

4.2. The Lacker Model. I give a short presentation of the result given in the paper of H.M. Lacker and A. Percus [21]. Here, a follicle is represented by its estradiol production $s_{i}$ which satisfies a conservation equation

$$
\frac{d}{d t} s_{i}=g_{i}\left(s_{i}, h_{1}, h_{2}\right),
$$

where $h_{1}$ represents the FSH concentration and $h_{2}$ the LH concentration. Since the FSH and LH production depends on the total production of estradiol of all the $N$ follicles : $\sum_{j=1}^{N} s_{j}$, we have

$$
\frac{d}{d t} s_{i}=g_{i}\left(s_{i}, \sum_{j} s_{j}\right)
$$

The assumption of H.M. Lacker and A. Percus was to consider that $g_{i}$ as the following form

$$
g_{i}\left(s_{i}, \sum_{j} s_{j}\right)=s_{i} F\left(\sum_{j} s_{j}\right)\left[G\left(\sum_{j} s_{j}\right)+\zeta\left(s_{i} / \sum_{j} s_{j}\right)\right]
$$

This is a mathematical assumption which leads to the following theorem

THEOREM 4.3. Under the assumption (4.9), we have that $y_{i}:=\sqrt{s_{i} / \sum_{j} s_{j}}$ satisfies

$$
\lim _{t \rightarrow \infty} y_{i}(t)=a_{i}
$$


where $a_{i}=0$ or $a_{i}=1 / \sqrt{M}$ and $M \in[1, N]$. Therefore, the dynamic of the system is quite simple (gradient system) and the authors are able to give the number of survival follicles independently of the initial data $s_{i}(0)$.

Even in the reduced model, a follicle is represented by its maturity (estradiol production) and its mass and the dynamic of the follicular system is much more complicated. In particular, the dynamic depends on the initial data of the mass and maturity of the follicles. Moreover, in the reduced model appears bifurcations and catastrophes, for instance a pool of follicles can disappear (atresia), and the more mature and big follicle at the beginning time $(t=0)$ is not necessarily the one which survives. This kind of dynamic, which are biologically observed, cannot be obtained by the H.M. Lacker model.

5. Discussion and perspectives. In this paper, we have studied the dynamics of a complex nonlinear partial differential system of equations. Using the Wasserstein distance, we have shown that the cell density concentrates its mass which means that the cells in a follicle have their maturity which become uniform. Then, we reduce the PDE system to an ODE system (always nonlinear). The dynamic of the "simpler" system is hard to study. Nevertheless, when we focus on a follicle, we are able to show that appears bifurcations (catastrophes) in its dynamic with taking in account the pressure of the others follicles (on this follicle) as an exogenous parameter. Then, we see that a follicle evolution is done in tree steps : first the follicle grows, second the follicle is sensitive to the pressure of the other follicle, finally if the follicle reaches the saved zone (not sensitive to the pressure) the important parameter is the mass the follicle acquired in the proliferation zone (first step).

The dynamic in this reduced model is much more complex than the dynamic of the Lacker model and is closer to the biology applications. In a future work, we will focus on finding the number of surviving follicles as it is possible to do in the Lacker model.

We have shown, see remark 4, that the injection of FSH improve the stability of the system but considering that injecting high level of FSH is dangerous for the body, we are interested to improve, efficiently, the number of follicles by injecting exogenous FSH.

Finally, we will see the impact of the variation of some parameters (in the definition of the local and global feedback control [14]) on the dynamic of the follicles and on the number of surviving follicles.

In a future work, we will focus on the explanation of biological observation on follicles evolution through the model proposed by INRIA team Sisyphe [14] we have studied here. For instance, with the help of biologists and specialists of folliculogenesis $([28,5,6])$, we will study the problems such as poly-kistic case where we observe large number of non-mature follicles without ovulation (see fig. 5.1), polyovulation (see fig 5.2), hormonal problems (FSH and LH) and their impact on the growth and maturation of follicles and finally to improve the understanding of the dynamic of follicle growth during the folliculogenesis. 
Mi

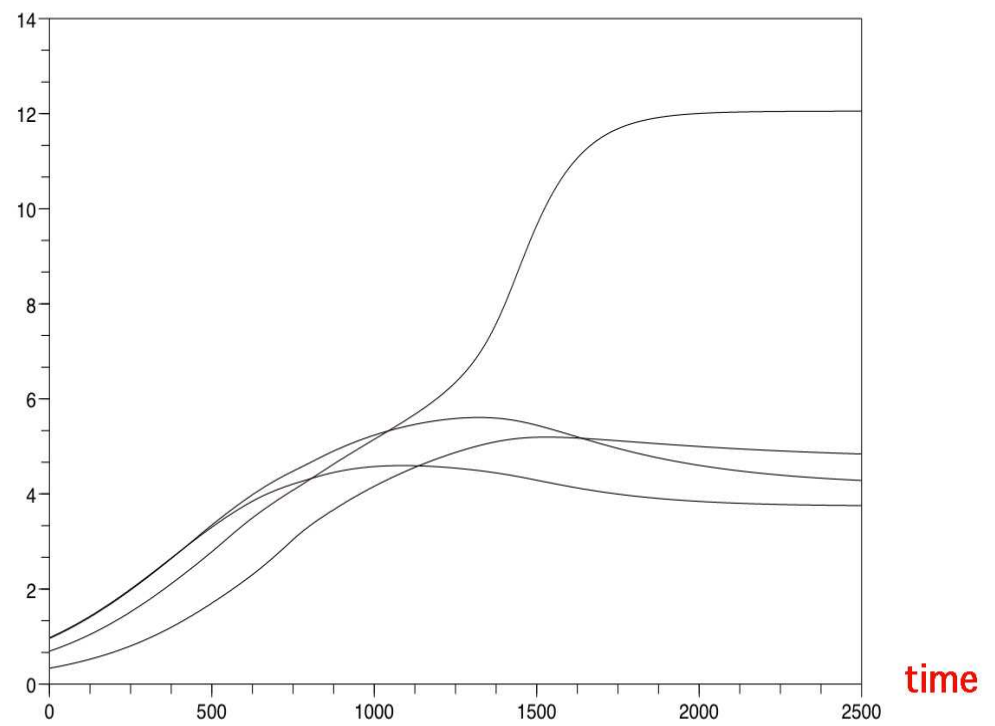

FIG. 5.1. Simulation: The follicle which has the better maturity at the end is not necessarily the one which have is the more mature at the beginning $t=0$.

Moreover, here we see that none of the follicle reach the ovulation threshold

(poly-kistic).

\section{REFERENCES}

[1] V.I. Arnold, Catastrophe Theory. (Translated by R. K. Thomas), Springer-Verlag, 79 pp, 1984.

[2] S-N Chow, J.K. Hale, Methods of bifurcation theory. A Series of Comprehensive Studies in Mathematics, vol. 251, Springer-Verlag, New York, 1982.

[3] Clement F., Multiscale modelling of endocrine systems: new insight on the gonadotrope axis. ESAIM: Proc., Vol. 27, pp. 209-226, 2009.

[4] F. Clement. Optimal control of the cell dynamics in the granulosa of ovulatory follicles. Mathematical Biosciences, 152, 123-142, 1998.

[5] F. Clement, M.A. Gruet, P. Monget, M. Terqui, E. Jolivet, D. Monniaux. Growth kinetics of the granulosa cell population in ovarian follicles : an approach by mathematical modelling. Cell Proliferation, 30, 255-270, 1997.

[6] F. Clement, D. Monniaux, J.C. Thalabard, D. Claude. Contribution of a mathematical modelling approach to the understanding of ovarian function. C. R. Biologies, 325, 473-485, 2002.

[7] J.M. Cushing, An Introduction to structured population dynamics. Philadelphia : SIAM, 1998.

[8] M. Demazure, Catastrophes et bifurcations, Ellipses, 1989.

[9] R.J. DiPerna, Measure valued solutions to conservation laws, Arch. Rational Mech. Anal., 88, p223-270, 1985.

[10] R.J. DiPerna and P.L. Lions, Ordinary differential equations, transport theory and Sobolev spaces, Invent. Math., 98, p511-547, 1989.

[11] R.J. DiPerna and P.L. Lions, Solutions globales dequations du type Vlasov-Poisson, C.R. Acad. Sci. Paris Sr. I, 307, p655-658, 1988.

[12] N. Echenim, Modelisation et controle multi-echelles du processus de selection des follicules ovulatoires, Phd Thesis, Universit Paris Sud-XI, 2006.

[13] N. Echenim, D. Monniaux, M. Sorine, F. Clement, Multi-scale modeling of the follicle selection process in the ovary, Math Biosci.,198(1), p57-79, 2005. 


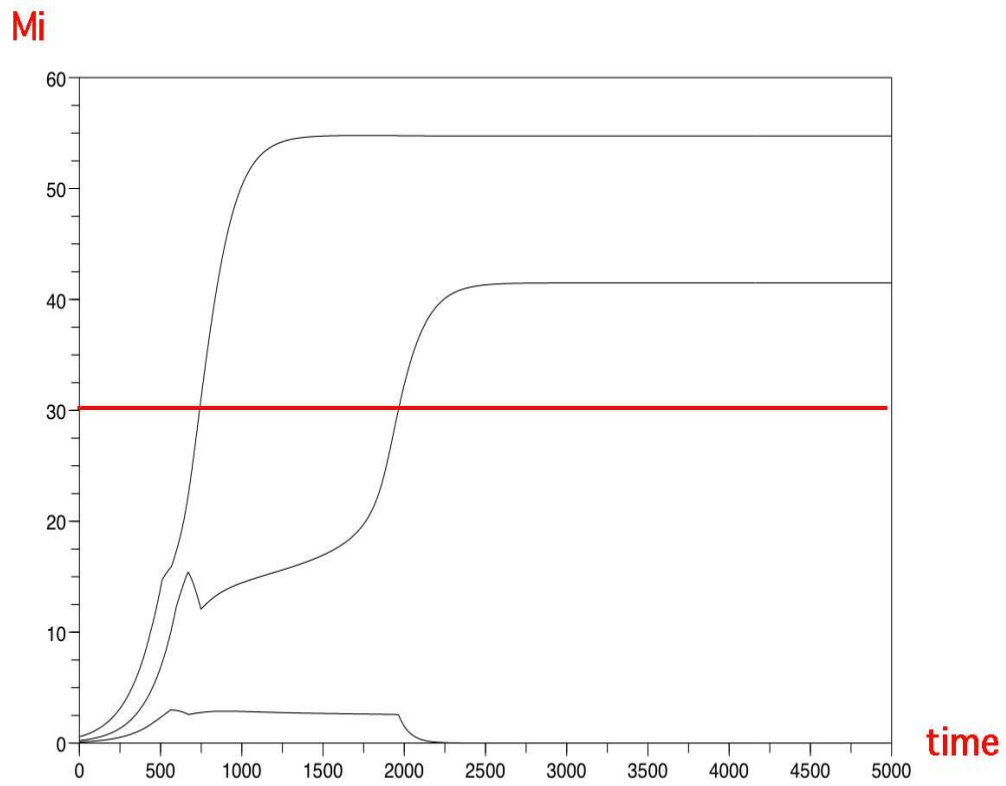

FIG. 5.2. Simulation: The number of survival follicles depends on the initial data (here this number is equal to 2).

[14] N. Echenim, F. Clement, M. Sorine. Multi-scale modeling of follicular ovulation as a reachability problem., Multiscale Modeling and Simulation, 6(3), 895-912, 2007.

[15] L.C. Evans, Weak Convergence Methods for Nonlinear Partial Differential Equations, American Mathematical Society, 1990. Third printing, 2002.

[16] R. Gilmore, Catastrophe Theory for Scientists and Engineers, Dover, New York, 1981.

[17] M. Iannelli, Age-Structured Population. In Encyclopedia of Mathematics, supplement II. Hazewinkel M. (a cura di), : Kluwer Academics, pp. 21-23, 2000.

[18] M. Iannelli, Mathematical theory of age-structured population dynamics, Applied Mathematics Monograph C.N.R., Vol. 7, In Pisa: Giardini editori e stampatori, 1995.

[19] P.E. Jabin, Macroscopic limit of Vlasov type equations with friction, Ann. IHP Anal. Non Lineaire, 17, p651-672, 2000.

[20] P.E. Jabin, Large time concentration for solutions to kinetic equations with energy dissipation, Comm. Partial. Differential Equations, 25, p541-557, 2000.

[21] H.M. Lacker and A. Percus, How do ovarian follicles interact? A many-body problem with unusual symmetry-breaking properties, J. Stat. Phys. 63, 11331161, 1991.

[22] V. Lewis, Reproductive Endocrinology and Infertility, Publisher: Landes Bioscience, Inc., 2007.

[23] T. Lundy, P. Smith, A. O'Connell, N. L. Hudson and K. P. McNatty, Populations of granulosa cells in small follicles of the sheep ovary, Journal of Reproduction and Fertility, 115, 251$262,1999$.

[24] E.A. McGee and A.J. Hsueh, Initial and cyclic recruitment of ovarian follicles. Endocrine reviews, 21(2), 200-14, 2000.

[25] P. Michel, A singular asymptotic behavior of a transport equation, Comptes Rendus Mathematique, 346, Issues 3-4, 155-159, 2008.

[26] P. Michel, S. Mischler, and B. Perthame. General relative entropy inequality: an illustration on growth models. J. Math. Pures Appl. (9), 84(9):12351260, 2005.

[27] S. Mischler, B. Perthame and L. Ryzhik, Stability in a Nonlinear Population Maturation Model, Mathematical Models and Methods in Applid Sciences, 12 No. 12, p1-22, 2002.

[28] D. Monniaux et al., Développement folliculaire ovarien et ovulation chez les mammifères, Inra Prod. Anim., 22 (2), 2009 (to appear).

[29] F. Otto and M. Westdickenberg, Eulerian Calculus for the contraction in the Wasserstein 
distance, SIAM J. Math.Anal., 37, p1227-1255, 2005.

[30] B. Perthame. Transport equations in biology. Frontiers in Mathematics. Birkhauser Verlag, Basel, 2007.

[31] B. Perthame. Mathematical tools for kinetic equations. Bul l. Amer. Math. Soc. (N.S.), 41(2), 205244 (electronic), 2004.

[32] B. Perthame. The general relative entropy principle applications in Perron-Frobenius and Floquet theories and a parabolic system for biomotors. Rend. Accad. Naz. Sci. XL Mem. Mat. Appl. (5), 29(1), 307-325, 2005.

[33] T. Poston, I. Stewart, Catastrophe Theory and its Applications, Dover, New York, 1978.

[34] M. Rotenberg, Transport theory for growing cell populations, J. Theor. Biol., 103, 181-199, 1983.

[35] R. Thom, Structural stability and morphogenesis. (Translated by D. H. Fowler 1975) Benjamin, Reading, Mass, 1972.

[36] J.A. Visser and A.P. Themmen, Anti-Mullerian hormone and folliculogenesis. Mol Cell Endocrinol, 234, 81-86, 2005.

[37] G.F. Webb, Theory of Nonlinear Age-dependent Population Dynamics, Pure and Applied Mathematics, vol. 89, Marcel Dekker, New York, 1985. 